\title{
Toxicity and Biodegradation of Zinc Ferrite Nanoparticles in Xenopus laevis
}

M. Rivero ${ }^{1 *}$, M. Marín-Barba ${ }^{2}$, L. Gutiérrez ${ }^{3}$, E. Lozano-Velasco², G. N. Wheeler², J. Sánchez-Marcos ${ }^{1}$ A. Muñoz-Bonilla ${ }^{4}$, C. J. Morris ${ }^{5}$, and A. Ruiz ${ }^{5 \star *}$

\author{
${ }^{1}$ Synthesis and Electrochemical and Spectroscopic Characterization of Materials Group, Departamento \\ de Química-Física Aplicada, Facultad de Ciencias, Universidad Autónoma de Madrid, C/Francisco \\ Tomás y Valiente 7, 28049 Madrid, Spain. \\ ${ }^{2}$ School of Biological Sciences, University of East Anglia, Norwich Research Park, Norwich, UK. \\ 3Departmento de Química Analítica, Instituto de Ciencia de Materiales de Aragón (ICMA/CSIC), \\ Universidad de Zaragoza and CIBER-BBN, 50018, Zaragoza, Spain. \\ ${ }^{4}$ Macromolecular Engineering Group, Instituto de Ciencia y Tecnología de Polímeros (ICTP-CSIC), \\ C/Juan de la Cierva 3, 28006 Madrid, Spain. \\ ${ }^{5}$ School of Pharmacy, University of East Anglia, Norwich Research Park, Norwich, UK. \\ tPresent address: School of Pharmacy, Queen's University Belfast, 97 Lisburn Rd, Belfast, BT9 7BL, \\ UK. G.RuizEstrada@qub.ac.uk \\ ¥These authors contributed equally to this work.
}

\section{Abstract}

$\mathrm{Zn}$-doped $\mathrm{Fe}_{3} \mathrm{O}_{4}$ magnetic nanoparticles have been proposed as the ideal ferrite for some biomedical applications like magnetic hyperthermia or photothermal therapy because of the possibility to adjust their size and chemical composition in order to design tailored treatments. However, reliable approaches are needed to risk assess $\mathrm{Zn}$ ferrite nanoparticles before clinical development. In this work, the in vitro toxicity of the nanoparticles was evaluated in five cellular models (Caco-2, HepG2, MDCK, Calu-3 and Raw 264.7) representing different target organs/systems (gastrointestinal system, liver, kidney, respiratory system and immune system). For the first time, these nanoparticles were evaluated in an in vivo Xenopus laevis model to study whole organism toxicity and their impact on iron and zinc metabolic pathways. Short and long-term in vivo exposure studies provided insights into the contrasting adverse effects between acute and chronic exposure. Quantitative PCR combined with elemental analysis and $A C$ magnetic susceptibility measurements revealed that at short-term exposure $(72 \mathrm{~h})$ the nanoparticles' absorption process is predominant, with the consequent over-expression of metal transporters and metal response proteins. At long-term exposure $(120 \mathrm{~h})$, there is an up-regulation of 
metal accumulation involved genes and the return to basal levels of both iron and zinc transporters, involved in the uptake of metals. This suggests that at this stage the nanoparticles' absorption process is residual compared with the following steps in metabolism, distribution and/or excretion processes, indicated by the increase of iron accumulation proteins at both transcriptional and translational level. This testing approach based on a battery of cellular systems and the use of the Xenopus laevis model could be a viable strategy for studying the toxicity, degradability and ultimately the long-term fate of zinc ferrites in the organism.

Keywords: zinc ferrite nanoparticles, Xenopus laevis, toxicity, biodegradation, metabolism.

\section{Introduction}

Due to the exponential growth of nanotechnology in recent decades and its wide range of applications, nanomaterials are already found in everyday products such as paints, building materials, cosmetics and in the food industry, exerting a great impact on today's society (Vance et al., 2015; Chaurasia, 2017; Mrowiec, 2017). Unfortunately, the rate of nanotechnological development has exceeded the rate at which we understand the toxicological risks to human health and the environment (Blaise et al., 2009; Jain et al., 2018; Lei et al., 2018).

The large surface area:volume ratio results in an increase in the reactivity of nanoparticles (NPs). Importantly, this affects the mechanisms of interaction with biological systems and these are far from being completely elucidated due to their complexity (Hagens et al., 2007). Even NPs of the same material can show different toxicities due to, for example, small differences in the size, shape, degree of aggregation, coating or surface charge (Arora, Rajwade and Paknikar, 2012). In addition, as soon as the NPs come into contact with the biological environment, the adsorption of biomolecules and the formation of the so-called "protein corona" begins, modifying the properties of the NPs and making their behaviour difficult to predict (Dai et al., 2018). Finally, the degree of toxicity varies depending on the dose of NPs used and the exposure time (Lewinski, Colvin and Drezek, 2008; Webster et al., 2016).

Magnetic iron oxide NPs constitute one of the most promising systems in the field of biomedicine. Their low toxicity, biocompatibility, excellent magnetic properties (superparamagnetism) and easy and versatile surface functionalization, open a wide range of possible biomedical applications. These applications cover diagnostic functions as contrast agents in magnetic resonance imaging ( $\mathrm{Na}$, Song and Hyeon, 2009), biosensors (Hasanzadeha, Shadjou and de la Guardia, 2015) or cellular labelling (Tefft et al., 2015), as well as therapeutic functions, such as magnetic hyperthermia (Laurent et al., 2011), controlled drug release (Zhang et al., 2017), tissue regeneration (Gonçalves, Rodrigues and Gomes, 2017) or gene therapy (Cheong et al., 2009). Recent advances in nanomedicine have led to the development of intelligent nanomaterials, combining both diagnosis and therapeutic functions, providing synergistic effects known as "theragnostics" (Hajba and Guttman, 2016). 
Spinel ferrites are good candidates for these purposes. Specifically, zinc ferrite $\left(\mathrm{ZnFe}_{2} \mathrm{O}_{4}\right) \mathrm{NPs}$, or zinc doped magnetite $\left(\mathrm{Zn}_{x} \mathrm{Fe}_{3-\mathrm{x}} \mathrm{O}_{4}(0<\mathrm{x}<1)\right) \mathrm{NPs}$, stand out against the rest of ferrites due to their favourable chemical and thermal stability (Raeisi-Shahraki et al., 2012) and their reduced toxicity (Wan et al., 2012). Since $\mathrm{Fe}$ and $\mathrm{Zn}$ are involved in a large number of biological processes, it is predicted that they can be safely incorporated in the labile pools of both elements (Kruszewski and Iwaneńko, 2003; Thambiayya et al., 2012). In addition, this system has distinctive magnetic properties due to the nonmagnetic nature of the zinc atom, which makes their modification and improvement possible simply by tuning the chemical position on the NPs (Liu et al., 2016).

The potential use of zinc ferrite NPs in biomedicine requires an in-depth study of the possible toxicity induced by NPs administration. Previous studies have reported the induction of chromosomal aberration in the meristematic root sunflower cells (Foca-Nici, Capraru and Creanga, 2010), the generation of cyto- and genotoxicity in human amnion epithelial (WISH) cell line (Saquib et al., 2013), cytotoxicity and oxidative stress in different human cells (Alhadlaq, Akhtar and Ahamed, 2015), as well as antioxidant activity (Kanagesan et al., 2016). However, the lack of consensus in the experimental conditions (methods, materials, and cell lines used) makes it difficult to obtain edifying conclusions. Also, nanotoxicological assessment should be accompanied by a thorough characterization of the physicochemical properties of the material. This highlights the need to create standardized nanotoxicity protocols in order to utilise the promising biomedical applications offered by NPs.

Furthermore, it is not uncommon to obtain contradictory results in vitro and in vivo, given the large number of biological processes that occur in the body which are difficult to reproduce in vitro. The early developmental vertebrate model Xenopus laevis has been recently proposed as a potential tool, able to bridge the gap between in vitro cell-based assays and mammalian models of toxicity assessment (Webster et al., 2016; Al-Yousuf et al., 2017; Mamusa et al., 2017; Marín-Barba et al., 2018; Saide, Sherwood and Wheeler, 2018; González-Paredes et al., 2019). The mortality, malformations and growth inhibition of Xenopus laevis have been studied, after exposure with metal oxide-based nanomaterials $\left(\mathrm{Y}-\mathrm{Fe}_{2} \mathrm{O}_{3}, \mathrm{TiO}_{2}, \mathrm{ZnO}\right.$ and $\mathrm{CuO}$ ) in a water contamination scenario (Nations, Long, et al., 2011; Nations, Wages, et al., 2011; Bacchetta et al., 2014; Perelshtein et al., 2015). Despite available literature on the effects of many compounds on the larval development of $X$. laevis in environmental studies, there are only few reports evaluating nanoparticles toxicity and biodistribution designed for biomedical applications, and there is still a lack of knowledge bridging biotransformation studies in cellbased assays with data generated from rodent in vivo systems in this area. Finally, the use of $X$. laevis larvae as a model is an important step towards facilitating a reduction of the number of mammals in pre-clinical research. We believe that the use of the Xenopus laevis model in combination with in vitro testing in mammalian cell lines constitutes an effective, quick and inexpensive tool to assess nanotoxicity prior to in vivo mammalian tests.

Public health risk assessments for nanomaterials must evaluate realistic NP exposure rates in order to draw meaningful conclusions from both in vitro and in vivo studies. A recent study evaluated the toxicity of $\mathrm{Zn}_{0.4} \mathrm{Fe}_{2.6} \mathrm{O}_{4} \mathrm{NPs}$ in mice after sub-chronic intragastric administration (50 mg per $\mathrm{kg} \mathrm{BW}$ of $\mathrm{Zn}_{0.4} \mathrm{Fe}_{2.6} \mathrm{O}_{4} \mathrm{NPs}$ for 30 consecutive days) (Zhu et al., 2016). Although exposure of high doses of NPs 
can lead to a rapid detection and quantification of the unsafe threshold, the main public health concern related with nanomaterials should refer to chronic low dose exposures throughout life (Elsaesser and Howard, 2012).

Several SPION formulations for intravenous or oral administration have been approved for clinical use as MRI contrast agents by the EMEA and FDA; however, the majority of the compounds that were approved for intravenous administration have, at present, been taken off the market (Estelrich, SánchezMartín and Busquets, 2015). Only the SPION for oral administration, Gastromark $®$ (AMAG Pharmaceuticals, Waltham, MA, USA; ferumoxsil, silicone-coated SPIONs), is currently on the market for gastrointestinal bowel marking. Notably, most contemporary NP toxicology studies focus on parenteral exposure of the NPs, while nanotoxicity following oral administration is under-reported. Ingestion of NP through food and drink consumption is likely the predominant route for oral exposure. However, chronic inhalational exposure would increase exposure through ingestion of NPs that have been deposited on the mucociliary escalator and cleared to the oropharynx.

Here, we report on the toxicity and biodegradation of $\mathrm{Zn}_{0.5} \mathrm{Fe}_{2.5} \mathrm{O}_{4} \mathrm{NPs}$ by means of a combination of studies using in vitro mammalian cell lines and in vivo Xenopus laevis embryos, which display spontaneous oral ingestion of NPs (Tussellino et al., 2015; Galdiero et al., 2017). To complement the in vivo system, in vitro studies include models of the major absorptive mucosal barriers of the lung and intestine as well as the excretory/clearance mechanisms present in the liver, kidney and the mononuclear phagocytic system. The in vivo test consisted of a comparative study of short and longterm exposures to the NPs to give insights about the effects between acute and chronic contact with the NPs. We consider that the time factor is of utmost importance in this analysis since many metal oxide NPs aggregate in exposure media and they may obstruct small vesicles and orifices, while some NPs that remain as single particles can translocate through membranes and reach critical compartments leading to intracellular alterations. Besides, the extent to which a metal oxide NP dissolves in the exposure medium or is biodegraded in the organism is an important consideration (Karimi et al., 2018). The expression patterns of both Fe and Zn metabolism related genes indicated that at short-term exposures $(72 \mathrm{~h})$ there is an activation of the elements' absorption by the organism, with the concomitant activation of the metal transporters and metal response proteins. At long-term exposures $(120 \mathrm{~h})$ these mRNA transcripts returned to basal levels, while there is an over-expression of the metal storage ones, highlighting the complete metal absorption and biodegradation of the nanoparticles.

The methodology reported here gives the opportunity to follow both the magnetic and structural transformations of the NPs along with their metabolic pathway. The combination of magnetic methods like AC susceptibility measurements with elemental analysis by Inductively Coupled Plasma-Optical Emission Spectrometry (ICP-OES) or elemental mapping by Scanning Electron Microscope with EDS elemental analyser along with more biological techniques like real time quantitative PCR, allow us to correlate the biodegradation of the magnetic nanoparticle, its bioaccumulation and the triggering of metabolic pathways involving $\mathrm{Fe}$ and $\mathrm{Zn}$ in the embryo. 


\section{Materials and Methods}

\section{Synthesis of magnetic NPs}

Zinc doped magnetite NPs, $\mathrm{Zn}_{0.5} \mathrm{Fe}_{2.5} \mathrm{O}_{4}$, with a diameter of $12 \mathrm{~nm}$ were prepared following an electrochemical synthesis method previously described (Rivero et al., 2016). Ferrite NPs are generated by the electro-oxidation of two iron and zinc metal foils (Goodfellow, 99.5\% and 99.9\% of purity, respectively) of $2 \mathrm{~cm}^{2}$ each, acting as anodes, placed parallel to each other in an electrochemical cell. A cylindrical iron counter-electrode with an area of $120 \mathrm{~cm}^{2}$ was employed as the cathode. The reaction was carried out in a $100 \mathrm{~mL}$ reactor containing an aqueous solution of $0.04 \mathrm{M}$ of tetrabutylammonium bromide ( $\left.\mathrm{nBu}_{4} \mathrm{NBr}\right)$ (Merck) acting as supporting electrolyte and surfactant at the same time. The selected zinc ferrite composition was obtained by applying $100 \mathrm{~mA}$ of current intensity to the iron anode and $20 \mathrm{~mA}$ to the zinc one. The reaction temperature was fixed at $40^{\circ} \mathrm{C}$ to obtain the desired NP diameter. The synthesis was performed under constant magnetic stirring for 30 minutes. The obtained powder was magnetically separated, washed repeatedly with distilled water and dried under vacuum at $60^{\circ} \mathrm{C}$ for $12 \mathrm{~h}$.

\section{Surface modification}

Firstly, the NPs were coated with dopamine molecules via catechol chemistry (Ye et al., 2011), obtaining an active surface with free amino groups available for further functionalization (Mazur et al., 2013). 20 $\mathrm{mg}$ of $\mathrm{Zn}_{0.5} \mathrm{Fe}_{2.5} \mathrm{O}_{4} \mathrm{NPs}$ were briefly dispersed in $3 \mathrm{~mL}$ of acetonitrile (Sigma-Aldrich) and $250 \mathrm{mg}$ of dopamine hydrochloride (Sigma-Aldrich) were added to the solution. The mixture was treated in an ultrasonic bath for 1 hour. The resulting black mixture was centrifuged and washed with distilled water several times and stored at $4{ }^{\circ} \mathrm{C}$.

Citric acid was coupled to the amino groups of the NPs' surface via an ethyl-3-(3-dimethylaminopropyl)carbodiimide/N-hydroxysuccinimide (EDC/NHS) mediated coupling reaction (Sahoo et al., 2013). For that purpose, $0.16 \mathrm{mmol}$ of citric acid was first dissolved in a volume of $10 \mathrm{~mL}$ of DMF/distilled water $(1: 1 \mathrm{v} / \mathrm{v})$, with a $\mathrm{pH}$ of 8 . Then, $0.32 \mathrm{mmol}$ of EDC and NHS were added to the solution. The $\mathrm{pH}$ was readjusted to 8 and the mixture was left in the dark for 4 hours under magnetic stirring, to allow for the citric acid activation. Finally, coupling was achieved by the dropwise addition of the aqueous nanoparticle suspension to the activated citric acid mixture and stirring for 12 hours in dark conditions. The resulting NPs were centrifuged and washed with distilled water several times and stored at $4{ }^{\circ} \mathrm{C}$.

\section{NPs characterization}

Iron and zinc concentrations were measured through inductively coupled plasma optical emission spectroscopy (ICP-OES) using a Perkin Elmer Optima 2100 DV system. The particle sizes and morphologies were determined by transmission electron microscopy (TEM) with a JEOL JEM 1010 operating at acceleration voltage of $100 \mathrm{kV}$. The size distributions were determined by manual measurement of more than 100 particles using Image J software. Colloidal properties were studied in a 
Zetasizer Nano S, from Malvern Instruments (UK). The hydrodynamic size was determined by Dynamic Light Scattering (DLS) and the $\zeta$ - Potential was measured as a function of $\mathrm{pH}$ at $25^{\circ} \mathrm{C}$, using $\mathrm{HNO}_{3}$ and $\mathrm{KOH}$ to change the $\mathrm{pH}$ of the suspensions at $0.5 \mathrm{mg} / \mathrm{mL}$. Hydrodynamic size is given as the intensityweighted mean. Magnetic characterization was performed using a Vibrating Sample Magnetometer (VSM) MLVSM9 MagLab 9T, Oxford Instruments. The magnetization hysteresis loops, $M(H)$, were measured at the VSM at $290 \mathrm{~K}$, applying magnetic fields up to $1 \mathrm{~T}$.

\section{Cell culture}

Caco-2, HepG2, MDCK, Calu-3, Raw 264.7 were obtained from American Type Culture Collection (Manassas, VA, USA) and stored in liquid nitrogen. Cells were cultured as mono-layers in Dulbecco's modified Eagle medium supplemented with $2 \%$ penicillin-streptomycin and $10 \%$ foetal bovine serum, in a humidified incubator $\left(37^{\circ} \mathrm{C}, 5 \% \mathrm{CO}_{2}\right)$. For toxicity experiments, cells were seeded in 96-well plates (approximately $1 \times 10^{4}$ cells/well, $200 \mu \mathrm{L} /$ well).

\section{Cytotoxicity assay (MTT)}

Cell viability was determined using the standard 3-(4,5-dimethylthiazol-2-yl)-2,5-diphenyl tetrazolium bromide (MTT) assay 24h after exposure to NPs. Cells were seeded in 96-well plates (approximately 1 $\times 10^{4}$ cells/well, $200 \mu \mathrm{L} /$ well). In total 60 wells were seeded per plate ( 6 rows $\times 10$ columns) as the outer wells were left empty to avoid errors due to evaporation. Each row was used as a replicate (6 wells/condition) and serial dilutions went across the columns of the plate. Cells were left to grow until $70-80 \%$ confluency. NPs-containing medium was removed after $24 \mathrm{~h}$, cells were rinsed three times with PBS and MTT solution in medium (final MTT concentration $50 \mu \mathrm{g} / \mathrm{mL}$ ) was added and incubated ( $2 \mathrm{~h}$, $37^{\circ} \mathrm{C}$ ). The MTT solution was removed without disturbing the cells, $0.2 \mathrm{~mL} /$ well of DMSO and 0.025 $\mathrm{mL} /$ well of Sorensen buffer were added, the plates were shaken gently to dissolve formazan crystals and the absorbance was read on a microplate reader at $550 \mathrm{~nm}$. Cell viability (\%) was calculated as $[(A / B) \times 100]$, where $A$ and $B$ are the absorbance of treated and control cells, respectively. Values represent mean \pm SD of 3 independent replicas of the experiment.

\section{In vivo test}

All experiments were performed in compliance with the relevant laws and institutional guidelines at the University of East Anglia. The research has been approved by the local ethical review committee according to UK Home Office regulations. Xenopus laevis embryo toxicity assays were carried out as described before (Webster et al., 2016). Briefly, adult females were primed with PMSG (Pregnant Mare's Serum Gonadotropin) and induced with Chorulon. Eggs were obtained manually and fertilized in a petri dish by adding male sperm (male testis incubated with $2 \mathrm{~mL} 1 \times$ MMR (Marc's modified ringers) (50 mM NaCl, $1 \mathrm{mM} \mathrm{KCl}, 0.5 \mathrm{mM} \mathrm{MgCl}$, $1 \mathrm{mM} \mathrm{CaCl}$, $2.5 \mathrm{mM} \mathrm{HEPES}, \mathrm{pH}$ 7.5) + $8 \mathrm{~mL}$ Foetal Bovine Serum). The fertilized embryos were dejellied via 2\% L-Cysteine $\mathrm{pH} 8$ for 7 minutes and then washed in 0.1x MMR. Embryos were plated in BSA-coated petri dishes covered with $0.1 \times$ MMR until they reached stage 38 . They were distributed in 24-well plates (7 embryos/well) in $0.1 \times$ MMR medium containing the nanoparticles. Non-treated embryos were used as the control. For short-term exposure experiments $(72 \mathrm{~h})$ embryos were left at $23^{\circ} \mathrm{C}$, whilst for long-term exposure experiments $(120 \mathrm{~h})$, they 
were left at $18{ }^{\circ} \mathrm{C}$ until they reached stage 45 in both cases. The mortality and the morphological changes of the embryos were recorded every $24 \mathrm{~h}$ until embryos reached stage 45 . To identify the morphological changes of the treated embryos, tadpoles were fixed at the appropriate stage with MEMFA (3.7\% Formaldehyde, 10\% MEM salts (MEM 1x: 0.1 M MOPS (3-(N-morpholino) propanesulfonic acid) $\mathrm{pH}=7.4,2 \mathrm{mM}$ EGTA, $1 \mathrm{mM} \mathrm{MgSO}_{4}, 3.7 \%$ Formaldehyde) in $0.1 \% \mathrm{v} / \mathrm{v}$ Diethyl pyrocarbonate (DEPC) $\mathrm{H}_{2} \mathrm{O}$ ) overnight at $4{ }^{\circ} \mathrm{C}$. After that, embryos were washed in Phosphate Buffered Saline Tween-20 (PBST) 1x and observed under the stereomicroscope.

\section{Scanning Electron Microscopy on tissue sections}

Embryos were dehydrated in a serial dilution of PBS-Methanol, 5 minutes each wash and then washed in $100 \%$ ethanol twice. To embed the embryos in wax, they were directly washed in a $65^{\circ} \mathrm{C}$ oven first in histoclear, then in 1:1 histoclear:wax and finally three washes in 100\% wax, 30 minutes to $1 \mathrm{~h}$ each wash. Then the embryos were placed in molds with wax. Embryos were sectioned using a microtome generating $10 \mu \mathrm{m}$ slices. Slices were then dried overnight and then dewaxed with two washes of histoclear followed by 5 minutes washes in a serial dilution of Ethanol-water. Once the samples were hydrated and dry, they were analysed with the Scanning Electron Microscopy (JEOL JSM 5900 LV Scanning Electron Microscope with INCA EDS elemental analyser).

\section{Iron and Zinc content analysis}

Groups of seven embryos were pooled. Animals were weighed and lyophilized 72 hours in a Telstar lyoquest lyophilizer and the iron content was measured by Inductively Coupled Plasma-Optical Emission Spectrometry (ICP-OES) in an Optima 2100 DV from Perkin Elmer. For acid digestion and elemental analysis, $300 \mu \mathrm{L}$ of $69 \% \mathrm{HNO}_{3}$ were added to the samples. The mixtures were heated to approximately $80^{\circ} \mathrm{C}$ for one hour. After this time, the solutions were allowed to cool down to RT and transferred to $20 \mathrm{~mL}$ volumetric flasks filled up to the mark with MilliQ water resulting in two individual dilutions for each sample, which were measured three times either by ICP-OES. Lyophilized samples were also kept for magnetic characterization. The sample manipulation was performed using disposable plastic material to avoid ferromagnetic contamination.

\section{Identification of NPs in tissues by AC Susceptibility}

Pools of freeze-dried tadpoles were placed into gelatine capsules for their magnetic characterization. AC susceptibility measurements were performed using a Quantum Design MPMS-XL SQUID magnetometer with an $A C$ susceptibility option. The measurements were performed with $A C$ amplitude of $0.41 \mathrm{Oe}$, at a frequency of $11 \mathrm{~Hz}$, in the temperature range between 1.8 and $300 \mathrm{~K}$.

\section{RNA extraction and quantitative PCR}

Groups of 7seven embryos were snap frozen in liquid nitrogen. RNA was extracted using High Pure RNA isolation kit (Roche) and $1 \mu \mathrm{g}$ of RNA was taken to synthesize cDNA using Maxima First Strand CDNA synthesis kit (Thermofisher). RT-PCR was performed using SYBR Green detection method. Primers were designed to target a conserved region present in the long and short copy of the $X$. laevis genes. gapdh was used as a control housekeeper gene. 


\begin{tabular}{|l|l|}
\hline Primers & Sequence 5' $-3^{\prime}$ \\
\hline$d m t 1 ~ F$ & cagaggatgaaacgcactca \\
\hline$d m t 1 R$ & atcctgccactgatccagac \\
\hline fth1 $F$ & tggagtaacaccctggaagc \\
\hline fth1 $R$ & aggatcaaccttgtcggatg \\
\hline tf $F$ & agaaagggcaagtgggtttt \\
\hline tf $R$ & tctggcaaagtgacaacagc \\
\hline znT1 F & cagaaagagcggccatagac \\
\hline znT1 $R$ & atgcgagttgccgtaattct \\
\hline hspa9 F & tggagggaaaacaagcaaag \\
\hline hspa9 $R$ & gttagtgaccgcctgtcgtt \\
\hline mt4 F & aaagctgctgttcctgctgt \\
\hline$m t 4 R$ & tgcttcccttttcacagtgg \\
\hline gadph $F$ & ctttgatgctgatgctggaa \\
\hline gadph $R$ & gaagaggggttgacaggtga \\
\hline
\end{tabular}

\section{Results and discussion}

\section{NPs synthesis and characterization}

The synthesis of zinc ferrite NPs with controlled size and composition was carried out following a singlestep electrochemical method. The stoichiometry of the material, $\mathrm{Zn}_{0.5} \mathrm{Fe}_{2.5} \mathrm{O}_{4}$, was determined by the ICP-OES analysis. TEM micrographs showed a quasi-spherical shape of the NPs (Figure 1a). The particle size was calculated from TEM micrographs analysis, by measuring at least 100 NPs, obtaining a value of $12 \pm 4 \mathrm{~nm}$ (mean $\pm \mathrm{SD}$ ). Colloidal properties of aqueous NP suspensions at $\mathrm{pH} 7$ were evaluated by means of DLS and $\zeta$-Potential (Figures $1 \mathrm{~b}$ and $1 \mathrm{c}$, respectively). The results showed that the NPs' surface is negatively charged due to the presence of the carboxylic groups of the citric acid molecules, with a $\zeta$ - Potential of $-30 \pm 4 \mathrm{mV}$ and a hydrodynamic diameter of $158 \mathrm{~nm}(\mathrm{PDI}=0.178$ ). Remarkably, the hysteresis loop (Figure 1d). presented the " $S$ " shape typical of superparamagnetic materials, making the NPs suitable for controlled magnetic manipulation 
A)

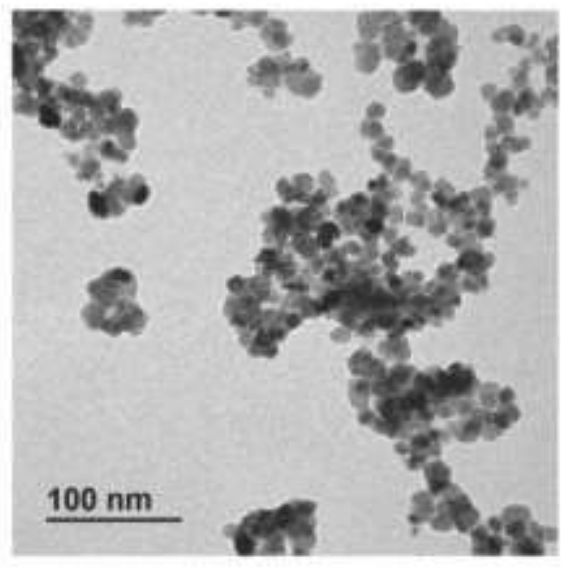

B)

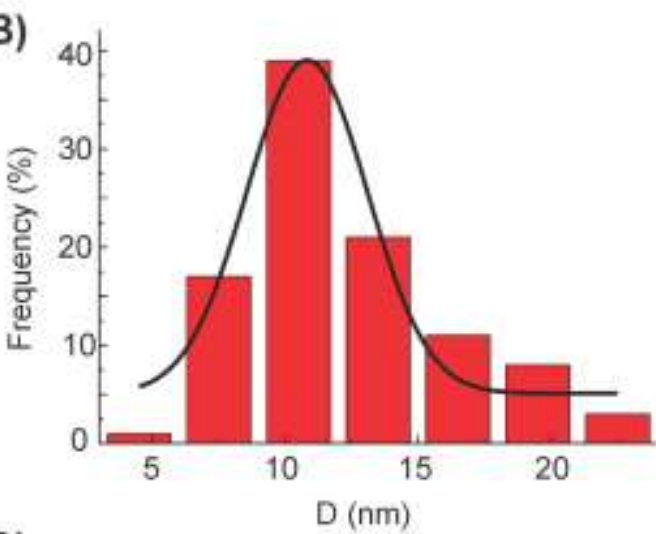

D)

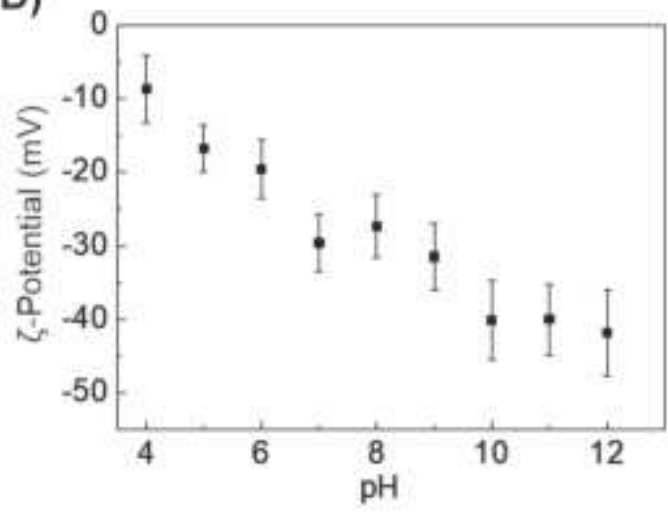

C)

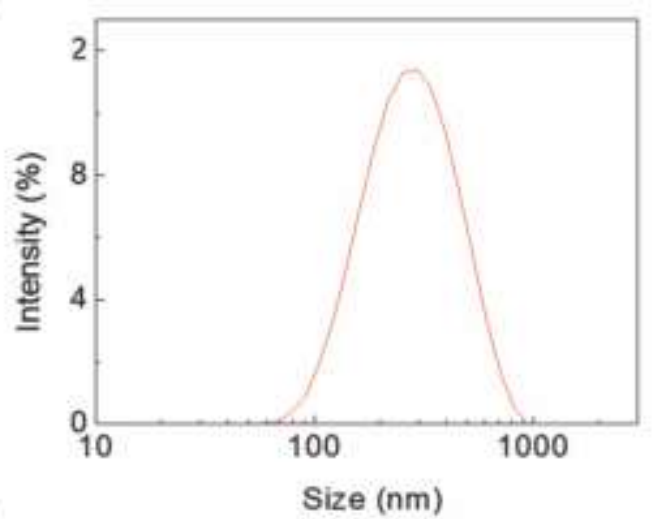

E)

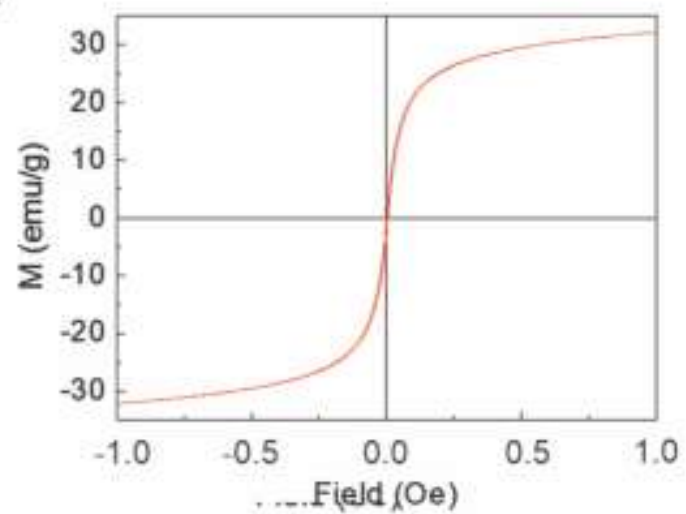

Figure 1. NPs characterization. A) TEM micrograph of the $\mathrm{Zn}_{0.5} \mathrm{Fe}_{2.5} \mathrm{O}_{4}$ NPs. B) Histogram of particle size distribution. C) Hydrodynamic size distribution of $\mathrm{Zn}_{0.5} \mathrm{Fe}_{2.5} \mathrm{O}_{4} \mathrm{NPS}$ obtained from DLS measurements of an aqueous NPs' solution at pH 7. D) $\zeta$ - Potential of an aqueous NP suspension as a function of $\mathrm{pH}$. E) Magnetic hysteresis loop of the NPs at $290 \mathrm{~K}$.

\section{In vitro toxicity}

Figure 2 summarizes the toxicological study in various cell lines, covering a broad spectrum of target organs/systems involved in the metabolism processes of the NPs after the oral exposure. The cells were incubated with the NPs for 24 hours in a range of concentrations between 0 to $1 \mathrm{mg} / \mathrm{mL}$ and the 
toxicological effect of the NPs was evaluated, by means of MTT assay. For the in vitro studies, the stock solution of NPs was diluted serially to yield concentrations ranging from $0.008 \mathrm{mg} / \mathrm{mL}\left(=5 \mu \mathrm{g} / \mathrm{cm}^{2}\right.$ microgram of particles sample to area of culture dish $\left.\left(\mu \mathrm{g} / \mathrm{cm}^{2}\right)\right)$ to $1 \mathrm{mg} / \mathrm{mL}\left(=6400 \mu \mathrm{g} / \mathrm{cm}^{2}\right)$. This study covers a wide range of concentrations to establish comparisons with different possible scenarios of in vivo exposure (low exposure vs. overloading) (Warheit, Sayes and Reed, 2009).

No evidence of toxicity was observed in the Calu-3 and Caco-2 cell lines, representing lung and intestine organs, respectively. Cell viability values remained around $100 \%$ in the whole range of concentrations studied. In the case of Hep G2 liver cell line, a slight decrease in viability was observed, with values between 80-90 \% across the concentration range. Therefore, it can be concluded that $\mathrm{Zn} 0.5 \mathrm{Fe}_{2.5} \mathrm{O}_{4} \mathrm{NPs}$ are potentially not toxic for these cell lines, at NPs' concentrations up to $1 \mathrm{mg} / \mathrm{mL}$, since cell viability values are maintained in the range of 80 to $100 \%$, in comparison with the control.

On the other hand, Raw 264.7 and MDCK cell lines, corresponding to macrophages and kidney cells, showed a progressive decrease in cell viability with the increase in NPs concentration, reaching minimum values of $72 \%$ and $55 \%$ at $1 \mathrm{mg} / \mathrm{mL}$ of NPs respectively. This result indicates that these NPs are moderately toxic to these types of cell lines, both of which would be involved in the clearance of NPs from the organism. The most sensitive cell line was MDCK, a well-known model for the renal distal tubular cells forming a very tight cellular barrier. These results are in agreement with other studies that show a significant dose-dependent cytotoxicity of iron oxide nanoparticles in this cell line. The generation of reactive oxygen species (ROS) by the nanoparticles is a major contributor to cytotoxicity. ROS formation, when it exceeds the cellular antioxidant defensive capacity, represented mainly by cellular thiols such as glutathione, induces oxidative damage to biomolecules, including lipids, proteins, and DNA, ultimately leading to cell death (Halamoda Kenzaoui, Chapuis Bernasconi and JuilleratJeanneret, 2013). Regarding Raw 264.7, macrophages constitute the central cellular component of the reticuloendothelial or mononuclear phagocytic system. Different studies have described how activated macrophages in presence of iron oxide nanoparticles generate ROS in a phenomenon called "oxidative burst" as part of the natural mechanism of defence of the immune system. Toxicity associated to the treatment with iron oxide nanoparticles was found to be due to induction of oxidative stress and subsequent apoptosis on concentrations above $100 \mu \mathrm{g} / \mathrm{mL}$ (Naqvi et al., 2010).

MTT assay offers a quick, inexpensive and high-throughput methodology to perform in vitro cytotoxicity screens. The experimental approach used in this study, involving multiple relevant cell lines, represents a way to avoid false-negative outcomes and obtained a more comprehensive view of zinc ferrites' biological activity. 


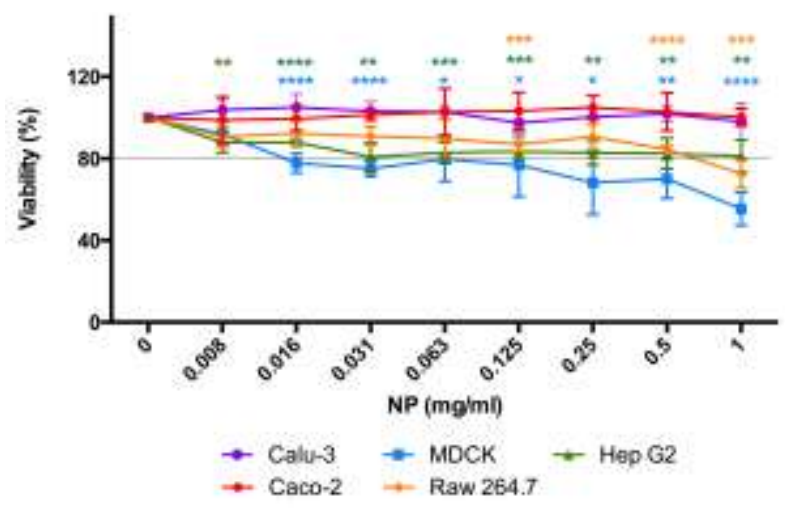

Figure 2. Evaluation of cell viability by MTT assay. Data represent means $\pm \operatorname{SD}(n=3)$. * shows statistically significance compared with the control (Student's t-test, ${ }^{*} p<0.05,{ }^{* *} p<0.01,{ }^{* * *} p<0.001$, $* * * * 00.0001)$.

\section{In vivo toxicity in Xenopus laevis}

We next sought to examine whether NP exposure via the oral route is likely to cause significant exposure to the major clearance organs - liver and kidney. Two different experiments were performed, exposing groups of $X$. laevis embryos at different concentrations of $\mathrm{Zn}_{0.5} \mathrm{Fe}_{2.5} \mathrm{O}_{4} \mathrm{NPs}$, specifically 0.5 , $1,1.5$ and $2 \mathrm{mg} / \mathrm{mL}$, leaving a group of untreated embryos as a control (Figure 3). In both experiments, the exposure of the embryos to the NPs was initiated in stage 38, just before the mouth opens, until reaching stage 45 , after the tadpole starts to ingest materials following a grazing behaviour, according to the development table of Nieuwkoop and Faber (Nieuwkoop and Jacob Faber, 1994). In Experiment 1 , the embryos were incubated at $23^{\circ} \mathrm{C}$, reaching stage 45 in approximately 72 hours. Experiment 2 was carried out at a lower temperature, $18{ }^{\circ} \mathrm{C}$, with the aim of delaying its development. In this way, stage 45 is reached in approximately 120 hours, which allowed for a study of longer exposure times to NPs while maintaining a consistent developmental stage. Long term (weeks' exposure) is not feasible if we aim to analyse the embryos at the same stage of organs development, therefore this was the best alternative. To determine the survival factor after the exposure to the NPs, dead embryo counts were made every 24 hours during the experiment.

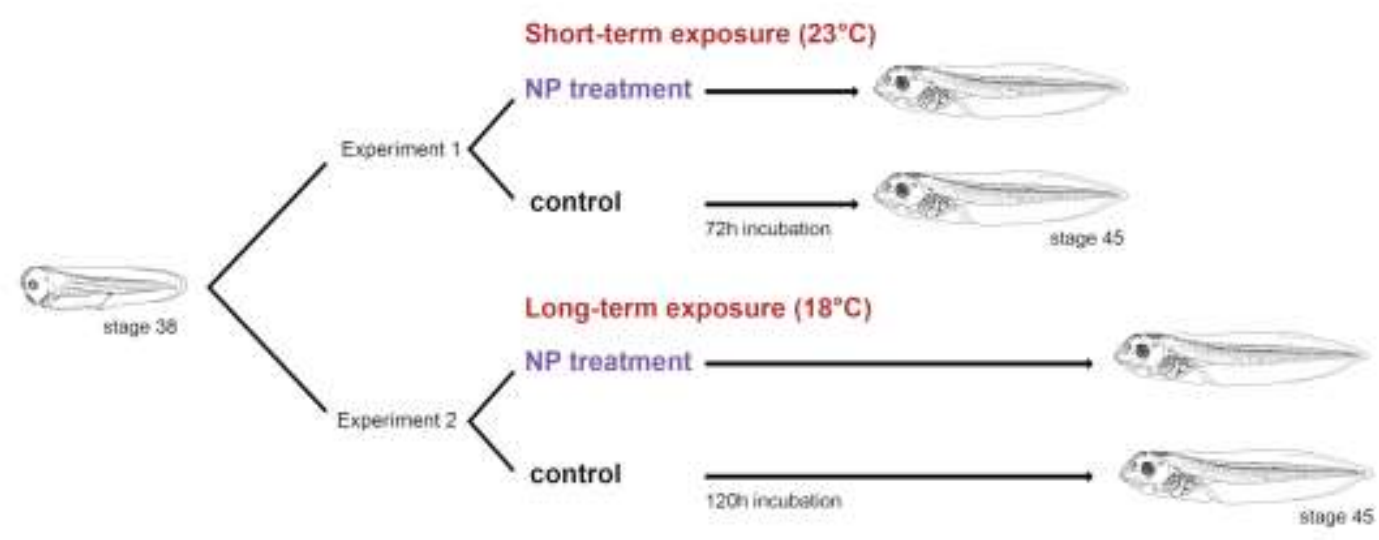


Figure 3. Schematic representation of exposure experiments to $\mathrm{Zn}_{0.5} \mathrm{Fe}_{2.5} \mathrm{O}_{4} \mathrm{NPs}$ carried out on $X$. laevis embryos.

Figure 4 shows the evolution of the mortality of both experiments, after 72 hours (Figure $4 a$ ) and after 120 hours of exposure (Figure 4b). No dead embryos were observed in the control groups of both short and long-term exposure experiments. In the short-term experiment (Figure 4a), no significant decrease in viability was observed after 48 hours for the different concentrations of NPs, except in the case of the highest concentration. At $2 \mathrm{mg} / \mathrm{mL}$, the survival factor reached $83 \%$ after 48 hours of exposure and only $46 \%$ after 72 hours. For the rest of experimental conditions, the NPs showed lower toxicity, increasing progressively as also does the NPs concentration and without the mortality rate exceeding $75 \%$.

For the long-term experiment (Figure 4b) similar results were obtained up to 72 hours. After 96 hours, a drastic increase in embryo mortality was detected in the case of the highest NPs concentration, 2 $\mathrm{mg} / \mathrm{mL}$, with only a $10 \%$ of survival factor at $96 \mathrm{~h}$. The NPs showed a moderate toxicity in the case of the $1.5 \mathrm{mg} / \mathrm{mL}$ condition, with a $70 \%$ of survival factor and low toxicity for 1 and $0.5 \mathrm{mg} / \mathrm{mL}$, increasing progressively as the NPs concentration rises. Finally, after 120 hours of exposure, significant mortality was observed in all cases, increasing the toxicity of NPs in a dose-dependent manner.
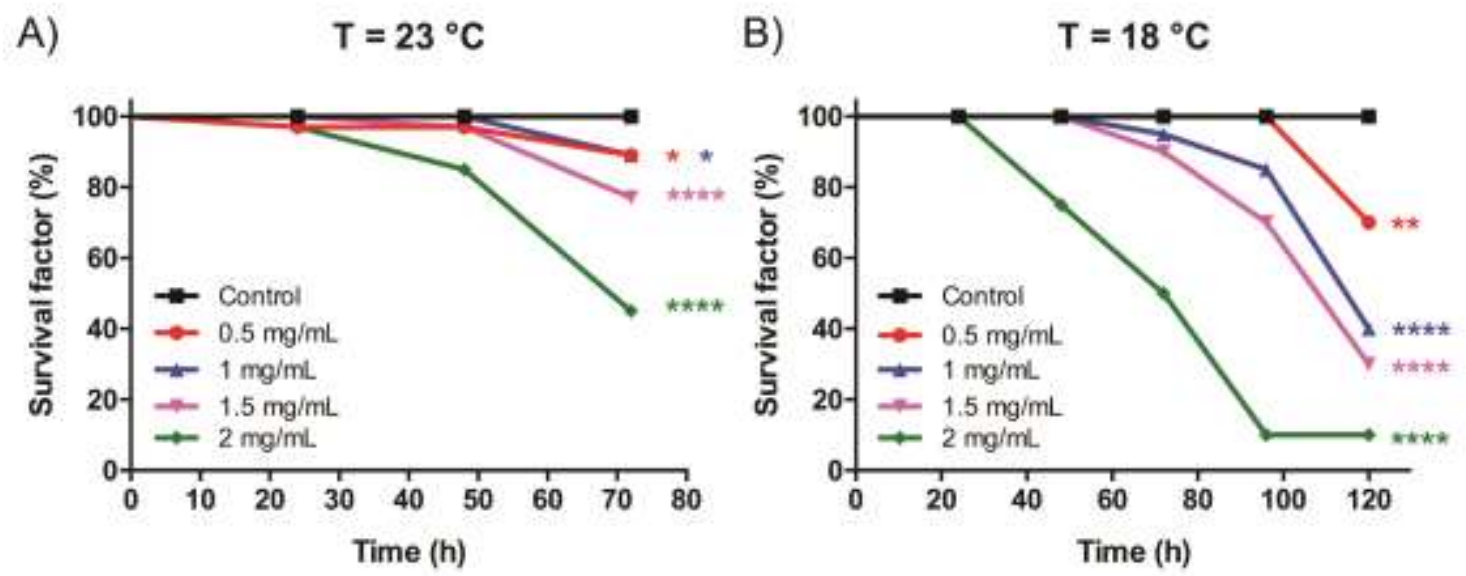

Figure 4. Survival factor of $X$. laevis embryos during the exposure experiments to the $\mathrm{Zn}_{0.5} \mathrm{Fe}_{2.5} \mathrm{O}_{4} \mathrm{NPs}$. A) Short-term exposure (72h) and B) Long-term exposure experiment (120h). * shows statistically significance compared with the control (Chi-square test, ${ }^{*} p<0.05,{ }^{* *} p<0.01,{ }^{* * *} p<0.001,{ }^{* * * *} p<0.0001$ ) ( $n=60$ per group).

Tadpoles displayed different abnormal phenotypes after exposure to the NPs, summarized in Figure 5a. These abnormalities consisted of impaired differentiation of the intestine visible as a swollen and deformed structure and enlarged ventral fin and bent spine. This delay in the intestine formation is suggested by the coiling process not being complete and the presence of vitelline platelets. These results are in agreement with other studies performing the exposure of embryos to $\mathrm{ZnO}$ NPs in two 
developmental windows (before and after stomodeum opening) which demonstrate that Xenopus laevis embryos become more susceptible to ZnO NPs with the acquisition of grazing behaviour following the stomodeum opening (Bonfanti et al., 2015). Figures $5 b$ and $5 c$ show the incidence in percentage of the different abnormal phenotypes observed after 72 hours and after 120 hours of experiment, respectively, on the surviving embryos. The results obtained in both experiments are comparable, reaching similar surviving rates after the same dose of NPs. This suggests that the effect is mainly due to the incubation with NPs and not due to the difference in temperature. Also, the surviving rate in both conditions is dose dependent (Figure 4). After $24 \mathrm{~h}$ of incubation with $2 \mathrm{mg} / \mathrm{mL}$ of NPs, the surviving rate of the embryos starts decreasing, reaching $40 \%$ at $72 \mathrm{~h}$ and drops to $10 \%$ at $120 \mathrm{~h}$ of incubation. In this last case, the mortality could be aggravated because the iron, which can catalyse the production of $\mathrm{H}_{2} \mathrm{O}_{2}$ and forming highly reactive hydroxyl radicals that result in mitochondrial dysfunction and oxidative damage, plays a key role at long term exposures.

In both experiments, impaired differentiation of the intestine, either moderate or severe, is observed in all of the experimental conditions, suggesting that the NP's are efficiently absorbed by the organisms triggering a delay in the intestine differentiation. Nevertheless, the decrease in the surviving rate is dose-dependent suggesting that despite that the absorption of the NPs triggering a delay in differentiation, in all experimental conditions the severity of the effects is proportional to the amount of NPs to which the embryos are exposed. Tadpoles with bent spine phenotype were detected in both experiments, without a clear trend associated with the dose of NP exposure. In addition, similar incidence of spine curvature was noted in the control tadpoles, preventing any causal links between this phenotype and NP toxicity being drawn. Tadpoles with enlarged ventral fins were only observed in the survivors of the short-term exposure experiment, increasing in incidence with the increase in the NPs concentration. A decrease in the incidence was observed for the highest concentration, although it was associated with higher mortality in that experimental condition. 
A)
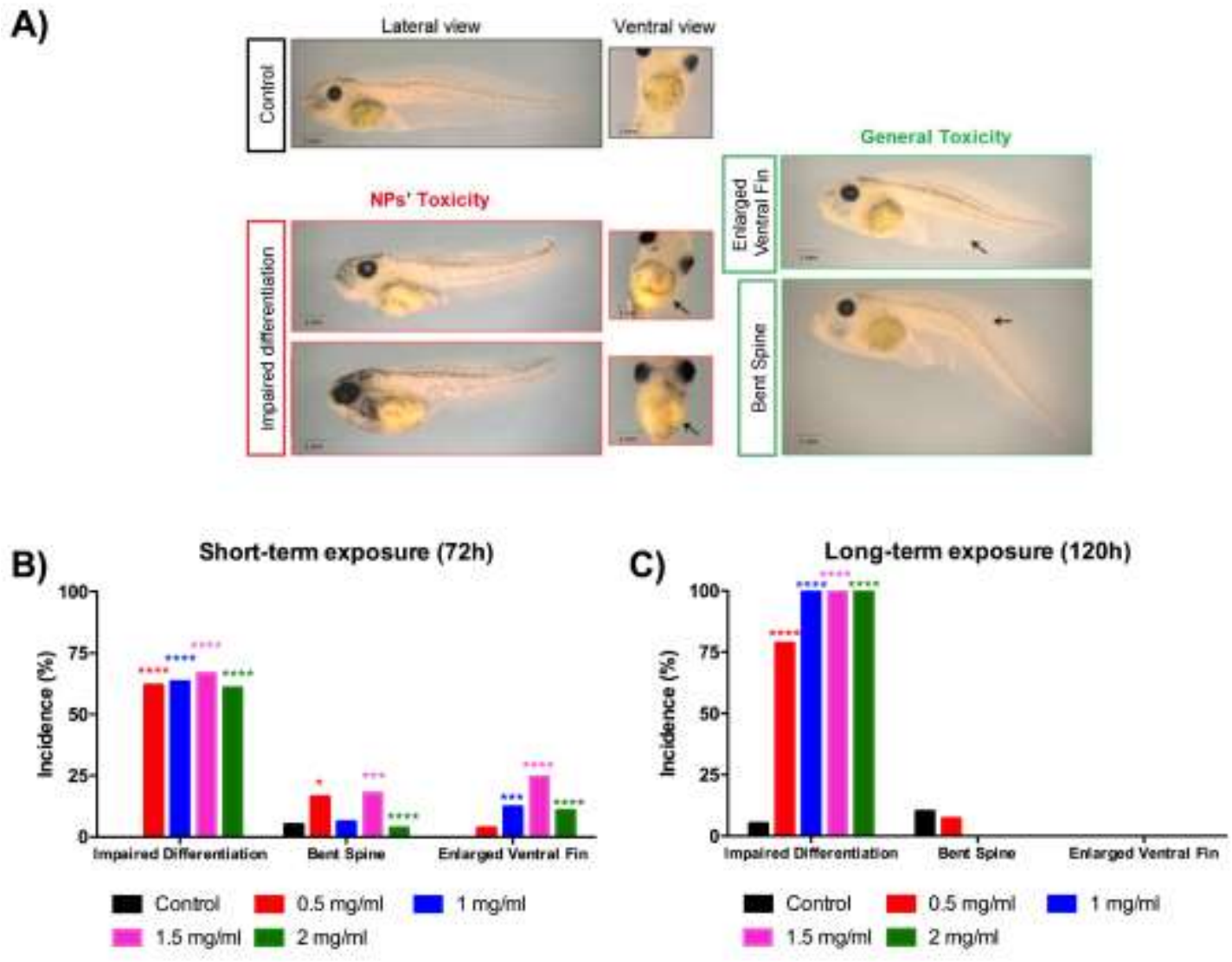

Figure 5. Xenopus laevis phenotypic nanotoxicity assay. A) Representative images of embryonic abnormalities. B) Quantification of the incidence of embryonic abnormalities over the surviving tadpoles after 72 hours and C) after 120 hours of NPs exposure. * shows statistically significance compared with the control (Chi-square test, ${ }^{*} p<0.05,{ }^{* *} p<0.01,{ }^{* * *} p<0.001,{ }^{* * *} p<0.0001$ ).

An elemental mapping analysis was carried out observing intestine and pharyngeal tissue sections of different embryos with a Scanning Electron Microscope (SEM) (Figure 6). The intestine and pharyngeal sections corresponding to the control embryos showed the normal/healthy morphology of both organs, with it being possible to distinguish the tubular structure of the intestine. The intestines of the treated embryos did not display the typical tube-shaped morphology indicating they have not undergone the cavitation process, thus suggesting a developmental delay as a consequence of NP exposure. The micrographs corresponding to pharynx sections of both embryos showed no morphological differences.

On the other hand, it was possible to detect the presence of spots with brighter contrast in the case of the NP exposed embryos, corresponding to areas of accumulation of NPs. The compositions of these spots were analysed by Energy Dispersive X-ray (EDS), evidencing the presence of Fe and $\mathrm{Zn}$ atoms in the elemental mapping and proving the effective intake of NPs by the embryos during the experiments. 


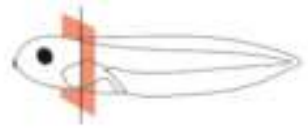

Intestine
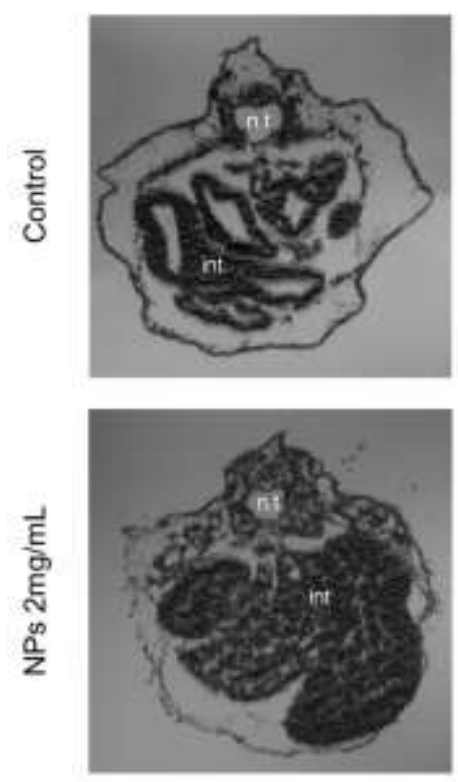

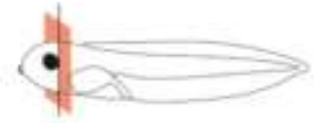

Pharynx
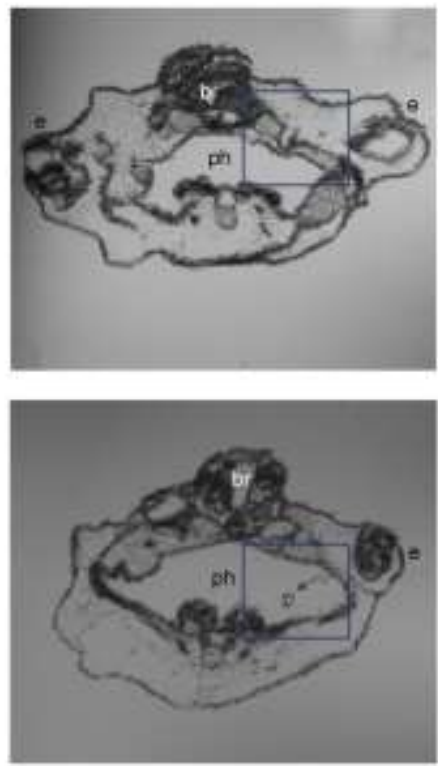

Elemental mapping
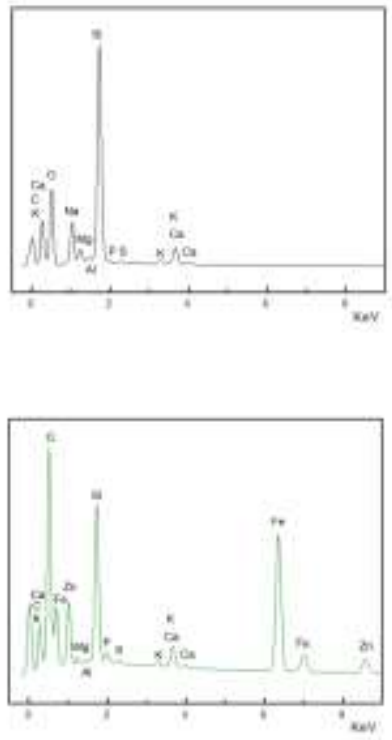

Figure 6. SEM micrographs of intestine and pharynx tissue sections of control and exposed embryos to a NPs concentration of $2 \mathrm{mg} / \mathrm{mL}$, after 72 hours. The elemental maps obtained by SEM-EDS, correspond to the selected areas (blue) in the images of the pharyngeal sections. $\mathrm{Ph}=$ pharynx, $\mathrm{br}=$ brain, e=eye, $n . t=$ neural tube, int $=$ intestine.

\section{Iron and Zinc quantification by ICP-OES}

A quantitative analysis of the NP intake by the embryos was performed by ICP-OES (Figure 7). In general, there is a clear increment in the Fe and $\mathrm{Zn}$ levels at short-term exposure, compared with the physiological levels, while reaching basal levels at long-term exposure. More specifically, higher amounts of both elements are detected at low NP doses $(0.5$ and $1 \mathrm{mg} / \mathrm{mL})$, decreasing the values afterwards, at short-term exposure. This could be explained as the embryos treated at higher doses of NPs have a less differentiated intestine and therefore the process of absorption is less effective. At long-term exposures, the same phenomenon is observed: the amount of metals detected is higher at lower dose than at higher dose. 

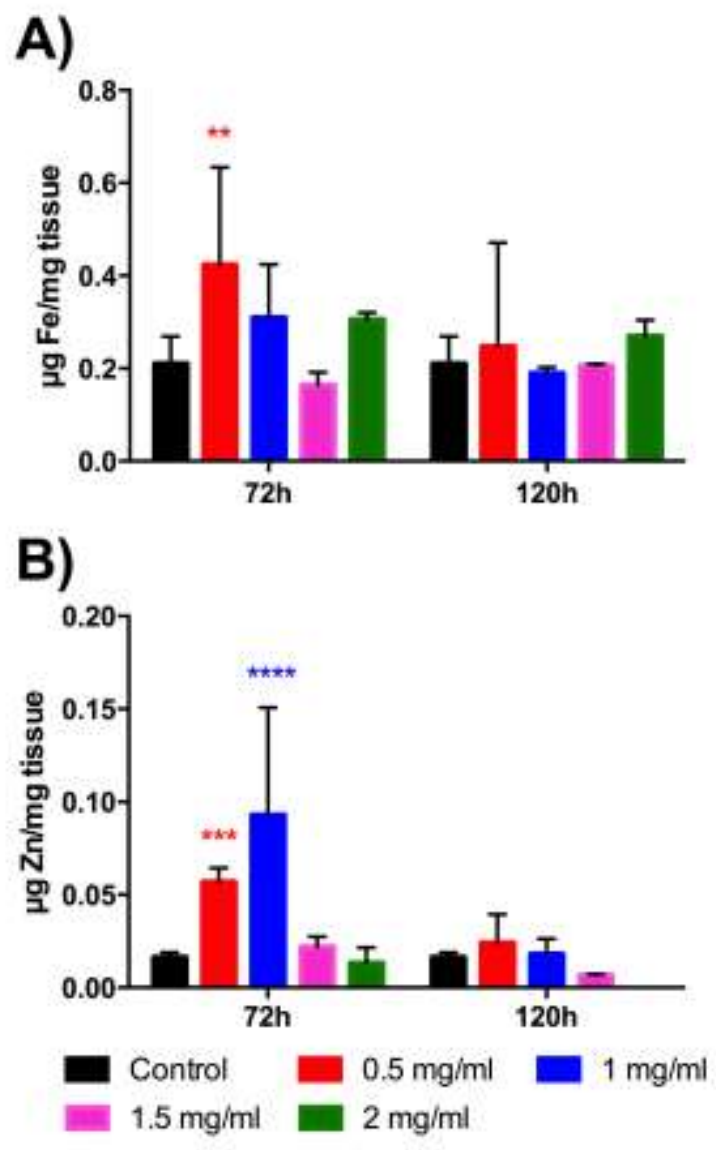

Figure 7. A) Iron and B) zinc quantification through ICP-OES of acid digested tissues from embryos treated at different concentrations of $\mathrm{Zn}_{0.5} \mathrm{Fe}_{2.5} \mathrm{O}_{4} \mathrm{NPs}$ ( $\mathrm{n}=7$ per group). * shows statistically significance compared with the control (Student's t-test, ${ }^{*} p<0.05$, ${ }^{* *} p<0.01,{ }^{* * *} p<0.001,{ }^{* * *} p<0.0001$ ).

\section{Metabolism and Biodegradation}

Due to the crucial role of both $\mathrm{Fe}$ and $\mathrm{Zn}$ elements in different cellular processes, the organisms present diverse mechanisms for their transport and storage in a non-toxic way (Zhang, Ghosh and Rouault, 2014), (Bell and Vallee, 2009). Both elements are supposed to be processed by their metabolic pathways after the NPs' degradation. Thus, the expression analysis of some of the principal genes involved in Fe and $\mathrm{Zn}$ metabolism was carried out by real time quantitative PCR (RT-qPCR), allowing a comparative study of the expression levels at short and long-term exposures at different NPS concentrations. The expression levels of control organisms were analysed individually to determine the differences regarding temperature and/or exposure time. Figure $\mathrm{S} 1$ shows how mRNA levels for $d m t 1$, transferrin and $h s p 9$ have no significant differences between short and long-term experiments. In the case of ferritin, znt1 and $m t 4$ their expression is increased at $120 \mathrm{~h}$ of exposure. Nonetheless, values corresponding to treated organisms are normalized with their individual experimental control. 
Figure 8 shows mRNA expression levels for ferritin, dmt1 and transferrin, all of which are involved in $\mathrm{Fe}$ metabolism. The levels of ferritin remained constant at short-term exposures, while its expression was up-regulated at long-term exposures, up to 4 -fold, in a dose-dependent manner. The expression of $d m t 1$ and transferrin presented the opposite behaviour, being up-regulated at short-term exposures and returning to basal levels at long-term exposures. Specifically, dmt1 levels increased around 3-fold, regardless of the NPs concentration and transferrin levels raised up as the dose of NPs increased. The mechanisms underlying $d m t 1$ up-regulation are largely unknown. Different studies have described that Ferroportin and Dmt1 expression are not altered in IRP2-/- mice (IRP2 is the chief physiological iron sensor in the cytoplasm) (Jiang et al., 2010; Wilkinson and Pantopoulos, 2014). On the other hand, other authors have described that zinc upregulates DMT1 protein and its mRNA expression and iron uptake (Yamaji et al., 2001). These observations support our results where we observed the upregulation of the divalent transporter at short-term exposure simultaneously with the highest levels of zinc detected by ICP at this stage (Yamaji et al., 2001).

In a first step after the metals uptake, we observed an over-expression of the divalent metal transporter1 (DMT1). This is located in the apical membrane of the enterocytes and is the primary transporter of dietary iron inside the cell (Garrick et al., 2003). It is worth mentioning that the DMT1 transporter is not iron selective and due to the presence of $\mathrm{Zn}^{2+}$ in the NPs composition, the up-regulation of this gene can also be influenced by both of these elements. After transcytosing the cell, Fe exits via the ferroportin transporter located in the basolateral membrane of the enterocytes to be loaded by transferrin, a Fe transporter glycoprotein in plasma (Harrison and Arosio, 1996). The expression of transferrin was also up-regulated at short term NP exposures in a dose-dependent manner, allowing the iron transport in the bloodstream to reach different organs.

In contrast, the expression of ferritin, the main storage protein of Fe within cells (Harrison and Arosio, 1996), remained at basal levels at short-term exposure, indicating the incomplete iron metabolization at this stage. Its expression was increased at longer exposure times, in a dose-dependent manner. In that stage, the DMT1 and transferrin transporters expression levels were similar to the control. This behaviour indicates the complete absorption and metabolization of iron, with it being found stored in the form of ferritin in the organism or excreted after 120 hours. These results corroborate the uptake and excretion pattern observed by ICP-OES analysis (Figure 7a). 


\section{A) Short-term exposure}

ferritin

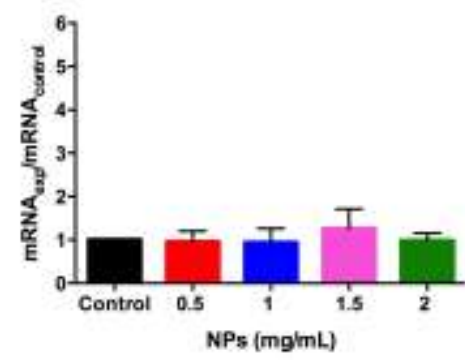

$d m+1$
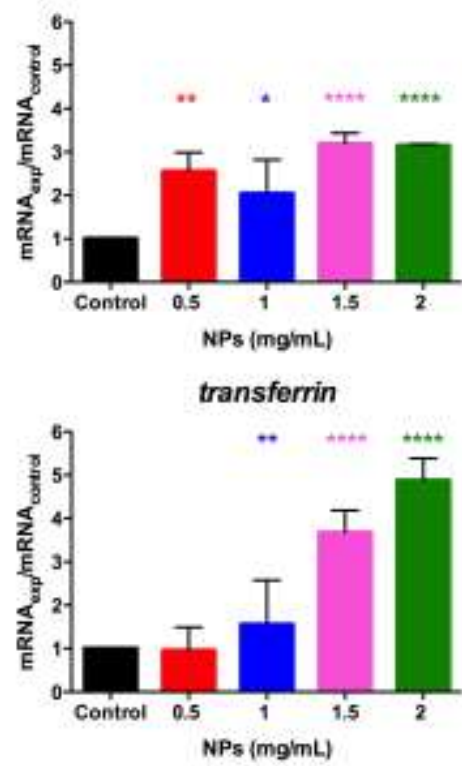

B) Long-term exposure

ferritin
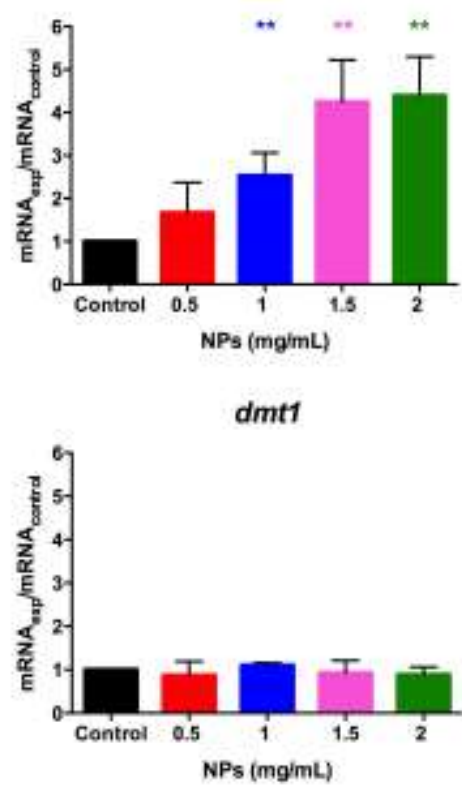

transferrin

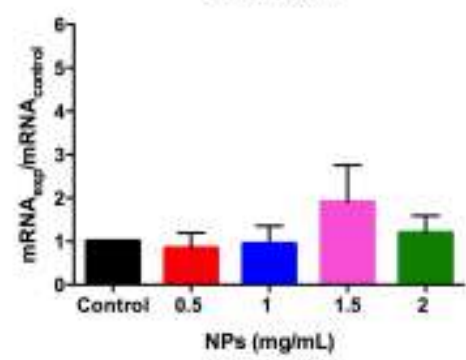

Figure 8. Effect on the expression of different genes involved in iron metabolism in $X$. laevis embryos exposed to different concentrations of $\mathrm{Zn}_{0.5} \mathrm{Fe}_{2.5} \mathrm{O}_{4}$ NPs after A) $72 \mathrm{~h}$ or B) $120 \mathrm{~h}$. ( $\mathrm{n}=7$ per group). * shows statistically significance compared with the control (Student's t-test, ${ }^{*} p<0.05,{ }^{* *} p<0.01$, $\left.{ }^{* * *} p<0.001,{ }^{* * *} p<0.0001\right)$.

Figure 9 shows the expression of $m t 4$, znt1 and hspa9 genes, involved in zinc metabolism. An overexpression of all genes was detected at short-term exposures, reaching the highest value of 5.3, 1.6 and 1.7 -fold at $1 \mathrm{mg} / \mathrm{mL}$ of NPs' concentration, in accordance with the ICP-OES peak value of $\mathrm{Zn}$ at this experimental condition (Figure 7b). Meanwhile, at higher concentrations the expression of those genes decreases slightly. This could be related with a delay/impairment in the development observed in the organ as a function of the dose. At long-term exposures, the expression of $m t 4$ is greatly increased, around 15 -fold for all the experimental conditions. The expression of znt 1 is only significantly increased, 2-fold, at $2 \mathrm{mg} / \mathrm{mL}$ NPs' concentration. In the case of the hspa9 gene, its expression kept basal levels in the whole range of NPs' concentrations.

$\mathrm{ZnT1}$ is a membrane transporter, belonging to the $\mathrm{ZnT}$ protein family, responsible for lowering the intracellular zinc by mediating zinc efflux from cells or influx into intracellular vesicles (Kambe, 
Hashimoto and Fujimoto, 2014). After zinc dietary absorption in the intestine through the Zip4 transporter, located in the apical membrane of the enterocytes (Cousins, Liuzzi and Lichten, 2006), $\mathrm{ZnT1}$, which appears to be the only $\mathrm{ZnT}$ transporter located at the plasma membrane, acts as the primary regulator of cellular zinc efflux (Palmiter and Findley, 1995). Its expression was up-regulated at $1 \mathrm{mg} / \mathrm{mL}$ NPs' concentration at short-term exposure, slightly decreasing the expression at higher NP doses, following a similar pattern as the ICP-OES results (Figure 7b). This behaviour could be explained by the activation of $\mathrm{Zn}$ metabolism and the beginning of the excretion process, with the consequent decrease of $\mathrm{Zn}$ levels in the organism. At long-term exposures, a significant increase in the gene expression was only detected at the highest NP concentration, indicating that the organism has almost finished the element excess excretion process.

Hspa9 is a mitochondrial $70 \mathrm{kDa}$ heat shock protein (mtHsp70), which is involved in cellular pathways regulating cell proliferation, tumorigenesis and the stress response (Wadhwa et al., 2003). It has been found to be highly expressed at the earliest stage of kidney development in Xenopus laevis (Gassié et al., 2015) and it's also regulated by the small zinc protein Zim17 (Vu et al., 2012). In our experiment, its expression seems to be regulated according to the $\mathrm{Zn}$ concentration levels, being over-expressed at short-time exposure and not induced at long-time exposure, corroborating the complete element excretion process at this stage.

Finally, MT4 expression was up-regulated in both short and long-term exposures, with a greater incidence in the last condition. MT4 belongs to the metallothionein family, which are low molecular weight proteins, rich in cysteine, involved in the regulation of the zinc homeostasis in the cytoplasm (Bonaventura et al., 2015). Its over-expression confirmed the effective zinc absorbance at the shortterm exposure stage 45 , reaching saturating values after long times of exposures. This result agrees with the up-regulation observed for ZnT1, promoting the zinc efflux for its clearance. 


\section{A) Short-term exposure}

znt1

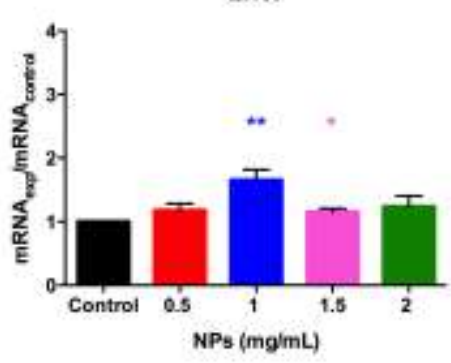

hspa9

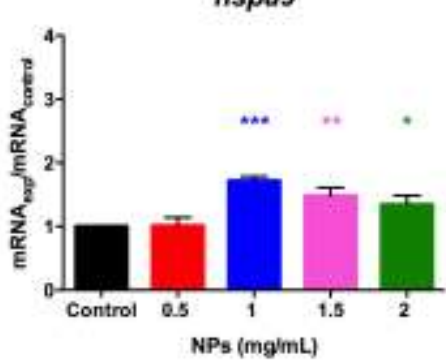

$m+4$

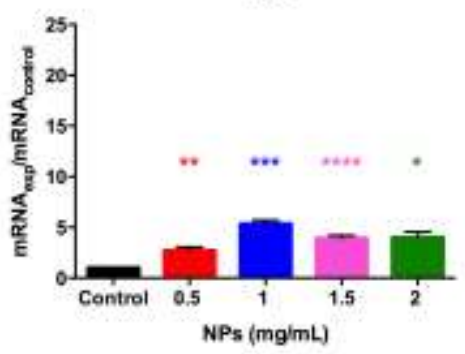

B) Long-term exposure

znt1

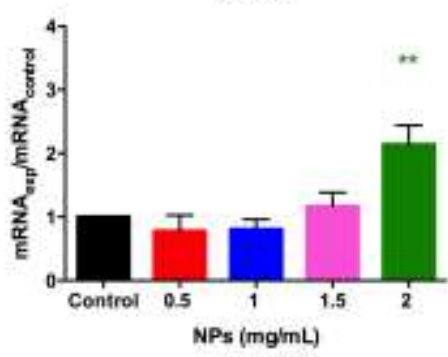

hspa9

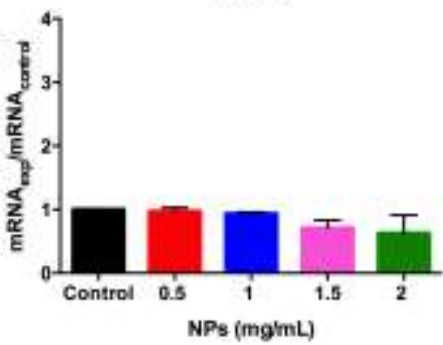

$m+4$

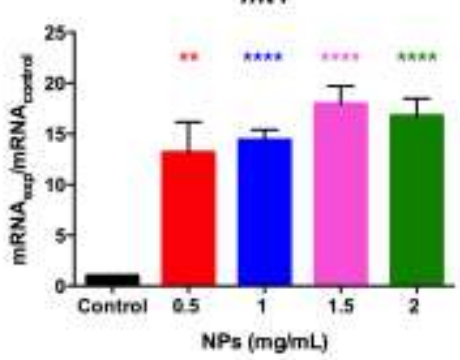

Figure 9. Effect on the expression of different genes involved in zinc metabolism in Xenopus laevis embryos exposed to different concentrations of $\mathrm{Zn}_{0.5} \mathrm{Fe}_{2.5} \mathrm{O}_{4} \mathrm{NPs}$ after A) $72 \mathrm{~h}$ or B) $120 \mathrm{~h}$. ( $\mathrm{n}=7$ per group). " shows statistically significance compared with the control (Student's t-test, ${ }^{*} p<0.05,{ }^{* *} p<0.01$, $\left.{ }^{* * *} p<0.001,{ }^{* * *} p<0.0001\right)$.

In summary, the expression patterns of both $\mathrm{Fe}$ and $\mathrm{Zn}$ metabolism related genes indicates that at short-term exposures (72h) there is an activation of the elements' absorption by the organism, with the concomitant activation of the metal transporters and metal response proteins. At long-term exposures $(120 \mathrm{~h})$ these proteins return to basal levels, while there is an over-expression of the metal storage ones, highlighting the complete metal absorption and biodegradation of the nanoparticles (Figure 10). The ICP-OES results indicate that both metal concentrations are close to basal levels as well, as a signal of the final stage metal excretion process at the time-scale of these experiments. In the case of the $\mathrm{Zn}$ metabolism, there is a partial overlap between absorption and storage processes at short-time exposures, suggesting that this metabolic pathway is faster than the Fe one. 


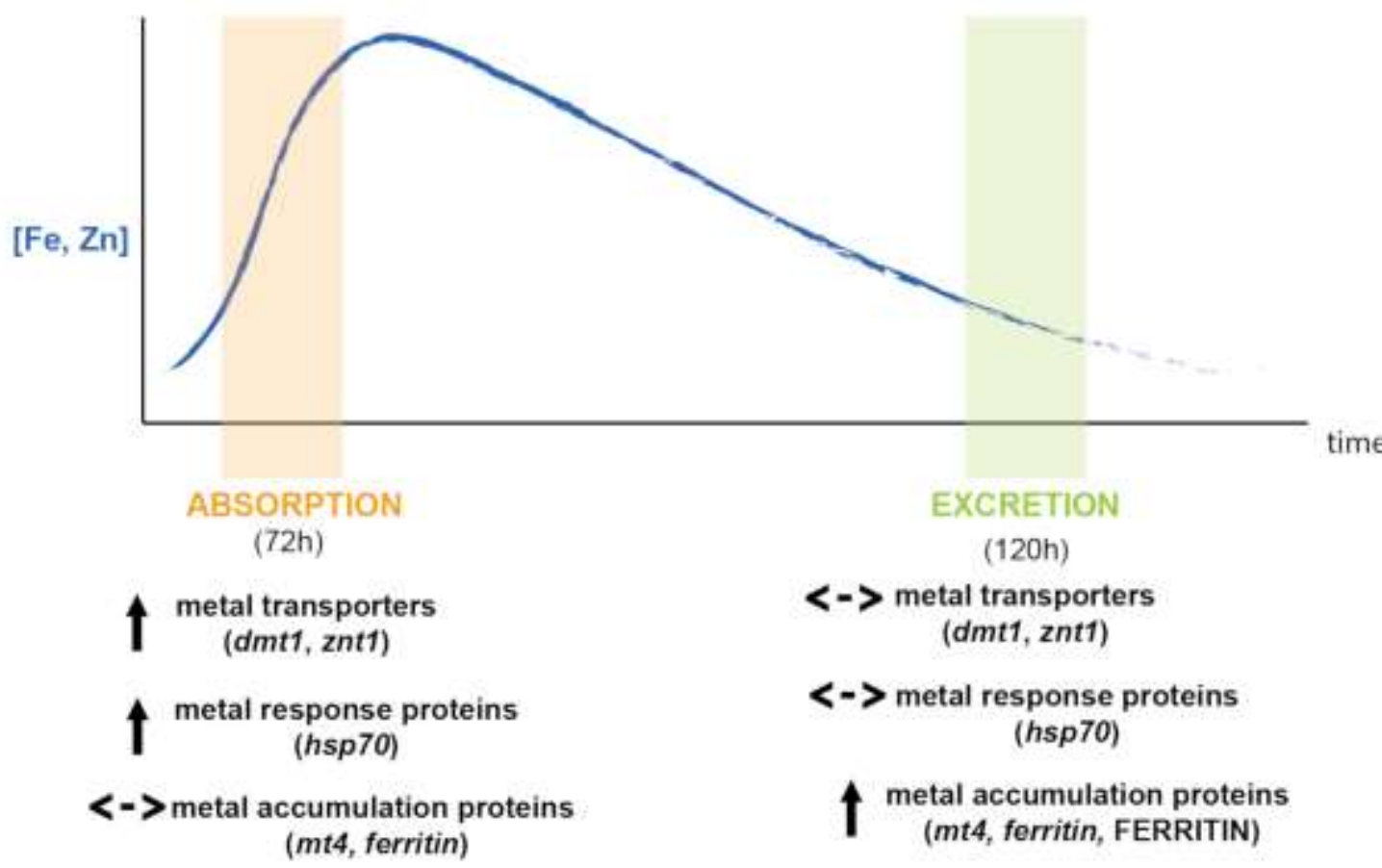

Figure 10. Schematic representation of the evolution of gene expression after NPs exposure with time.

\section{NPs accumulation}

With the aim of evaluating the accumulation of NPs in the tadpoles, AC magnetic susceptibility measurements were performed. This sensitive technique allows the discrimination between the magnetic NPs' contribution from that of other endogenous iron-containing species, usually present in a higher concentration but with a weaker magnetic signal.

A complex notation is used to describe the $\mathrm{AC}$ magnetic susceptibility time dependent behaviour: $\mathrm{X}_{\mathrm{AC}}=$ $X^{\prime}$ - ix", where $X^{\prime}$ and $X^{\prime \prime}$ are the in-phase susceptibility and out-of-phase susceptibility components respectively. In this case, an in-phase susceptibility increase with temperature, indicative of the presence of the particles, was observed only at short exposure times. As shown in Figure 11a, at long exposure times, only a weak diamagnetic signal (negative and constant in all the temperature range) can be observed in the in-phase susceptibility contribution, indicating that the presence of any remaining particle is below the detection limits of this technique. Interestingly, at the higher nanoparticle concentration $(2 \mathrm{mg} / \mathrm{mL})$ and the longest exposure time $(120 \mathrm{~h})$, a weak but measurable signal was observed at very low temperatures $(\approx 10 \mathrm{~K})$ (Figure $11 \mathrm{~b}$ ). The location in temperature of this signal agrees with previous measurements of ferritin, the iron storage protein, whose biomineralized core always presents a similar magnetic behaviour (independently of the animal species) when measured in the same conditions (Gutierrez et al., 2006, 2013; Cohen et al., 2010). These results are in agreement with ferritin over-expression pointed out by the RT-qPCR results (Figure 8b). 


\section{A)}

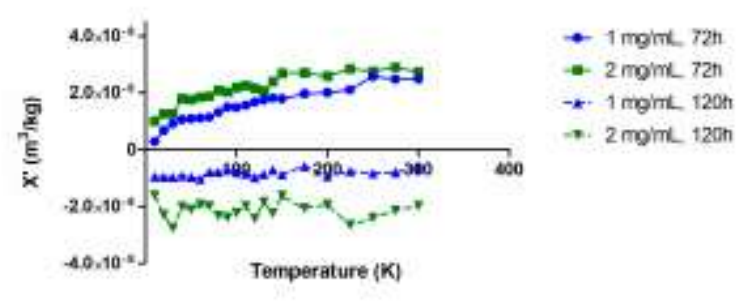

B)

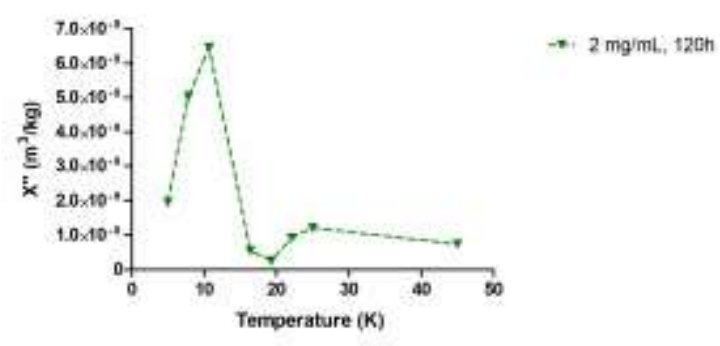

Figure 11. AC magnetic susceptibility measurement of embryo tissue samples. A) In-phase $\left(X^{\prime}(T)\right)$ and $B$ ) out-of-phase $\left(X^{\prime \prime}(T)\right)$ components of the magnetic susceptibility, per mass of sample, corresponding to pools of freeze-dried tissues from different embryos after short or long-term exposure (72 h or $120 \mathrm{~h}$ respectively). In blue embryos exposed to $1 \mathrm{mg} / \mathrm{mL}$ of $\mathrm{Zn}_{0.5} \mathrm{Fe}_{2.5} \mathrm{O}_{4} \mathrm{NPs}$. In green, Embryos exposed to $2 \mathrm{mg} / \mathrm{mL}$ of $\mathrm{Zn}_{0.5} \mathrm{Fe}_{2.5} \mathrm{O}_{4} \mathrm{NPs}$ ( $\mathrm{n}=7$ per group).

In this study, by means of RT-qPCR combined with elemental analysis and AC magnetic susceptibility measurements, we describe $\mathrm{Zn}$-doped $\mathrm{Fe}_{3} \mathrm{O}_{4}$ magnetic nanoparticles intake and metabolism in $X$. laevis embryos. We believe that physic-chemical characteristics of the nanoparticles in terms of structure and composition will dictate the time at which iron metabolic pathway activation is measurable.

These results correlate with previous work where iron oxide nanoparticle biodegradation was described in this amphibian model (Marín-Barba et al., 2018). Extensive quantitative studies of the long-term fate of iron oxide nanoparticles inside cells have been carried out. In situ monitoring in spheroids has described the degradation of the iron cores in the endosomes and revealed the loading of ferritin with free iron ions (Mazuel et al., 2016). Our approach reduces also the gap between amphibians and mammals. They are in correspondence with different studies in murine models tracking IONPS biodegradation. The significant increase of transcript levels of ferritin and the detection of the magnetic signal of ferritin correlates with previous observations in a murine model where the degradation of iron oxide nanocubes has been studied over 14 days after intravenous injection (Lartigue et al., 2013).

The presence of zinc in the structure of the nanoparticles seems to modulate the biodegradation and metabolism of the nanoparticles. Biodegradation of iron oxide nanoparticles triggered an iron metabolism response after $72 \mathrm{~h}$ with a 3-fold increase of ferritin levels, whilst with $\mathrm{Zn}$-doped $\mathrm{Fe}_{3} \mathrm{O}_{4}$ the 
over-expression of the metal storage protein takes place after $120 \mathrm{~h}$ of exposure. Also, for the first time we were able to detect, by AC magnetic susceptibility measurements in $X$. laevis, the presence of ferritin, highlighting the necessity of long-term studies to fully describe the degradability and biotransformation of these magnetic nanoparticles in the organism.

\section{Conclusions}

In this work, the toxicity and biodegradation of $\mathrm{Zn}_{0.5} \mathrm{Fe}_{2.5} \mathrm{O}_{4} \mathrm{NPs}$ by means of a combination of in vitro experiments using mammalian cell lines and in vivo experiments with the Xenopus laevis embryo model, after the oral administration of NPs, have been carried out. The in vitro study reveals that the NPs are not toxic to the Calu-3, Caco-2 and Hep G2 cell lines, maintaining the cell viability at concentrations of NPs up to the supraphysiological concentration of $1 \mathrm{mg} / \mathrm{mL}$. In contrast, the viability of Raw 264.7 and MDCK cell lines showed moderate toxicity at NPs concentration of $1 \mathrm{mg} / \mathrm{mL}$, indicating that they exerted more cytotoxic effect in cells lines involved in the NPs' clearance in the organism. These results are in line with the in vivo study: phenotypes related with general systemic toxicity (enlarged ventral fin or bent spine) are not significantly observed. Indeed, the main morphological effects (mild or severe impaired differentiation of tissues) occur in the gut, organ involved in the degradation and metabolism of the nanoparticles.

The in vivo survival factor revealed a progressive increase in the mortality rate with increasing NP concentrations and exposure times. Abnormal phenotypes associated with the NPs exposure were detected, mainly related to the gastrointestinal tract (swollen and deformed structure). Genetic expression patterns revealed that at short-term exposure $(72 \mathrm{~h})$, the NP absorption process is predominant, with the consequent over expression of metal transporters and metal response proteins. At long-term exposure (120 h), there is an up-regulation of genes involved in metal accumulation and a return to basal levels of both iron and zinc elements in the body, suggesting that at this stage, the NP absorption process is residual compared with processes such as metabolism, distribution and/or excretion. Depending on the structural composition of the nanoparticles $\left(\mathrm{Fe}_{3} \mathrm{O}_{4}\right.$ or $\left.\mathrm{Zn}_{0.5} \mathrm{Fe}_{2.5} \mathrm{O}_{4}\right)$, the embryos trigger the iron metabolic pathway to varying rates. The use of $X$. laevis model provides a rich source of data for determining nanoparticle toxicity and biodegradability in a quick an inexpensive way.

\section{Conflicts of interest}

There are no conflicts to declare.

\section{Acknowledgements}

M. Marín-Barba has been supported by the People Program (Marie Curie Actions) of the European Union's Seventh Framework Program FP7 under REA grant agreement number 607142 (DevCom). AR was supported by FP7-People Framework - Marie Curie Industry and Academia Partnerships \& 
Pathways scheme (DNA-TRAP project, grant agreement nr. 612338). L. Gutiérrez acknowledges financial support from the Ramón y Cajal subprogram (RYC-2014-15512). Servicio General de Apoyo a la Investigación-SAI, Universidad de Zaragoza is also acknowledged. E. Lozano-Velasco acknowledges support from Marie Curie fellowship (705089-MIR-CHROM-C). Authors acknowledge the facilities and the scientific and technical assistance, especially that of Bertrand Leze from the SEM service of the University of East Anglia.

\section{BIBLIOGRAPHY}

Al-Yousuf, K. et al. (2017) 'Combining Cytotoxicity Assessment and Xenopus laevis Phenotypic Abnormality Assay as a Predictor of Nanomaterial Safety', Current Protocols in Toxicology, 73:20(13.1-20), pp. 13-33.

Alhadlaq, H. A. H., Akhtar, M. J. M. and Ahamed, M. (2015) 'Zinc ferrite nanoparticle-induced cytotoxicity and oxidative stress in different human cells', Cell \& Bioscience. BioMed Central, 5, p. 55. doi: 10.1186/s13578-015-0046-6.

Arora, S., Rajwade, J. and Paknikar, K. (2012) 'Nanotoxicology and in vitro studies: the need of the hour', Toxicology and Applied Pharmacology. 2011/12/20, 258(2), pp. 151-165. doi: 10.1016/j.taap.2011.11.010.

Bacchetta, R. et al. (2014) 'Evidence and uptake routes for Zinc oxide nanoparticles through the gastrointestinal barrier in Xenopus laevis', Nanotoxicology, 8(7), pp. 728-744. doi: $10.3109 / 17435390.2013 .824128$.

Bell, S. G. and Vallee, B. L. (2009) 'The Metallothionein/thionein system: An oxidoreductive metabolic zinc link', ChemBioChem, 10(1), pp. 55-62. doi: 10.1002/cbic.200800511.

Blaise, C. et al. (2009) 'Ecotoxicity of Selected Nano-Materials to Aquatic Organisms', Environmental toxicology, 24(3), pp. 296-303. doi: 10.1002/tox.20402.

Bonaventura, P. et al. (2015) 'Zinc and its role in immunity and inflammation', 14(4), pp. 277-285. doi: 10.1016/j.autrev.2014.11.008.

Bonfanti, P. et al. (2015) 'Do Nanoparticle Physico-Chemical Properties and Developmental Exposure Window Influence Nano ZnO Embryotoxicity in Xenopus laevis?', Int. J. Environ. Res. Public Health International Journal of Environmental Research and Public Health ISSN, 123390, pp. 8828-8848. doi: 10.3390/ijerph120808828.

Chaurasia, N. (2017) 'Nanotechnology and Nanomaterials in Everyday Life', International Journal of Science and Research, 6(4), pp. 1560-1562.

Cheong, S. et al. (2009) 'Superparamagnetic iron oxide nanoparticles-loaded chitosan-linoleic acid nanoparticles as an effective hepatocyte-targeted gene delivery system', International Journal of 
Pharmaceutics, 8(372(1-2)), pp. 169-176.

Cohen, L. A. et al. (2010) 'Serum ferritin is derived primarily from macrophages through a nonclassical secretory pathway', Blood, 116(9), pp. 1574-1584. doi: 10.1182/blood-2009-11-253815.

Cousins, R. R. J., Liuzzi, J. J. P. and Lichten, L. L. A. (2006) 'Mammalian zinc transport, trafficking, and signals', 281(34), pp. 24085-24089. doi: 10.1074/jbc.R600011200.

Dai, Q. et al. (2018) 'Particle Targeting in Complex Biological Media', Advanced Healthcare Materials, 7(1), p. 1700575.

Elsaesser, A. and Howard, C. V. (2012) 'Toxicology of nanoparticles', Advanced Drug Delivery Reviews. Elsevier B.V., (2), pp. 129-137. doi: 10.1016/j.addr.2011.09.001.

Estelrich, J., Sánchez-Martín, M. and Busquets, M. (2015) 'Nanoparticles in magnetic resonance imaging: from simple to dual contrast agents', International journal of nanomedicine, 10, pp. 17271741.

Foca-Nici, E., Capraru, G. and Creanga, D. (2010) 'Comparative cytogenetic study on the toxicity of magnetite and zinc ferrite nanoparticles in sunflower root cells', AIP Conference Proceedings, 1311(2010), pp. 345-350. doi: 10.1063/1.3530036.

Galdiero, E. et al. (2017) 'Daphnia magna and Xenopus laevis as in vivo models to probe toxicity and uptake of quantum dots functionalized with gH625', International Journal of Nanomedicine. Dove Press, Volume 12, pp. 2717-2731. doi: 10.2147/IJN.S127226.

Garrick, M. D. et al. (2003) 'DMT1: A mammalian transporter for multiple metals', BioMetals, 16(1), pp. 41-54. doi: 10.1023/A:1020702213099.

Gassié, L. et al. (2015) 'Hspa9 is required for pronephros specification and formation in Xenopus laevis', 244(12), pp. 1538-1549. doi: 10.1002/dvdy.24344.

Gonçalves, A., Rodrigues, M. and Gomes, M. (2017) 'Tissue-engineered magnetic cell sheet patches for advanced strategies in tendon regeneration', Acta Biomaterialia, 63, pp. 110-122.

González-Paredes, A. et al. (2019) 'Solid lipid nanoparticles for the delivery of anti-microbial oligonucleotides', European Journal of Pharmaceutics and Biopharmaceutics. Elsevier, 134, pp. 166177. doi: 10.1016/J.EJPB.2018.11.017.

Gutierrez, L. et al. (2006) 'Bioinorganic transformations of liver iron deposits observed by tissue magnetic characterisation in a rat model', J Inorg Biochem. 2006/08/11, 100(11), pp. 1790-1799. doi: 10.1016/j.jinorgbio.2006.06.010.

Gutierrez, L. et al. (2013) 'Biophysical and genetic analysis of iron partitioning and ferritin function in Drosophila melanogaster', Metallomics. 2013/06/19, 5(8), pp. 997-1005. doi: 10.1039/c3mt00118k.

Hagens, W. et al. (2007) 'What do we (need to) know about the kinetic properties of nanoparticles in the body?', Regulatory Toxicology and Pharmacology, 49(3), pp. 217-229. 
Hajba, L. and Guttman, A. (2016) 'The use of magnetic nanoparticles in cancer theranostics: Toward handheld diagnostic devices', Biotechnology Advances, 34(4), pp. 354-361.

Halamoda Kenzaoui, B., Chapuis Bernasconi, C. and Juillerat-Jeanneret, L. (2013) 'Stress reaction of kidney epithelial cells to inorganic solid-core nanoparticles', Cell Biology and Toxicology, 29(1), pp. 39-58. doi: 10.1007/s10565-012-9236-8.

Harrison, P. M. and Arosio, P. (1996) 'The ferritins: Molecular properties, iron storage function and cellular regulation', Biochimica et Biophysica Acta - Bioenergetics, 1275(3), pp. 161-203. doi: 10.1016/0005-2728(96)00022-9.

Hasanzadeha, M., Shadjou, N. and de la Guardia, M. (2015) 'Iron and iron-oxide magnetic nanoparticles as signal-amplification elements in electrochemical biosensing', Trends in Analytical Chemistry, 72, pp. 1-9.

Jain, A. et al. (2018) 'Nanomaterials in food and agriculture: An overview on their safety concerns and regulatory issues', Critical Reviews in Food Science and Nutrition, 22(58(2)), pp. 297-317.

Jiang, H. et al. (2010) 'Up-regulation of divalent metal transporter 1 in 6-hydroxydopamine intoxication is IRE/IRP dependent', Cell Research. Nature Publishing Group, 20(3), pp. 345-356. doi: 10.1038/cr.2010.20.

Kambe, T., Hashimoto, A. and Fujimoto, S. (2014) 'Current understanding of ZIP and ZnT zinc transporters in human health and diseases', Cellular and Molecular Life Sciences, 71(17), pp. 3281 3295. doi: 10.1007/s00018-014-1617-0.

Kanagesan, S. et al. (2016) 'Evaluation of Antioxidant and Cytotoxicity Activities of Copper Ferrite (CuFe2O4) and Zinc Ferrite ( $\mathrm{ZnFe2O4)}$ Nanoparticles Synthesized by Sol-Gel Self-Combustion Method', Applied Sciences, 6(9), p. 184. doi: 10.3390/app6090184.

Karimi, S. et al. (2018) 'Acute and chronic toxicity of metal oxide nanoparticles in chemical mechanical planarization slurries with Daphnia magna', Environmental Science: Nano. Royal Society of Chemistry, 5(7), pp. 1670-1684. doi: 10.1039/c7en01079f.

Kruszewski, M. and Iwaneńko, T. (2003) 'Labile iron pool correlates with iron content in the nucleus and the formation of oxidative DNA damage in mouse lymphoma L5178Y cell lines', Acta Biochimica Polonica, 50(1), pp. 211-215.

Lartigue, L. et al. (2013) 'Biodegradation of iron oxide nanocubes: high-resolution in situ monitoring', ACS Nano, 28(7(5)), pp. 3939-3952.

Laurent, S. et al. (2011) 'Magnetic fluid hyperthermia: Focus on superparamagnetic iron oxide nanoparticles', Advances in Colloid and Interface Science. Elsevier B.V., 166(1-2), pp. 8-23. doi: 10.1016/j.cis.2011.04.003.

Lei, C. et al. (2018) 'Environmental transformations and ecological effects of iron-based nanoparticles', Environmental Pollution, 232, pp. 10-30. 
Lewinski, N., Colvin, V. and Drezek, R. (2008) 'Cytotoxicity of nanoparticles', Small. 2008/01/01, 4(1), pp. 26-49. doi: 10.1002/smll.200700595.

Liu, X. et al. (2016) 'Structural, Magnetic, and Thermodynamic Evolutions of Zn-Doped Fe3O4 Nanoparticles Synthesized Using a One-Step Solvothermal Method', The Journal of Physical Chemistry C, 120(2), pp. 1328-1341.

Mamusa, M. et al. (2017) 'Cationic liposomal vectors incorporating a bolaamphiphile for oligonucleotide antimicrobials', Biochimica et Biophysica Acta (BBA) - Biomembranes. Elsevier B.V., 1859(10), pp. 1767-1777. doi: https://doi.org/10.1016/j.bbamem.2017.06.006.

Marín-Barba, M. et al. (2018) 'Unravelling the mechanisms that determine the uptake and metabolism of magnetic single and multicore nanoparticles in a Xenopus laevis model', Nanoscale, 10(690-704). doi: 10.1039/C7NR06020C.

Mazuel, F. et al. (2016) 'Massive Intracellular Biodegradation of Iron Oxide Nanoparticles Evidenced Magnetically at Single-Endosome and Tissue Levels', ACS Nano, 10(8), pp. 7627-7638. doi: 10.1021/acsnano.6b02876.

Mazur, M. et al. (2013) 'Iron oxide magnetic nanoparticles with versatile surface functions based on dopamine anchors', Nanoscale, 5(7). doi: 10.1039/c3nr33506b.

Mrowiec, B. (2017) 'Nanomaterials in the environment', in International Conference on Advances in Energy Systems and Environmental Engineering (ASEE17). Wrocław, Poland, p. 22, id.00119. doi: 10.1051/e3sconf/20172200119.

$\mathrm{Na}, \mathrm{H}$., Song, I. and Hyeon, T. (2009) 'Inorganic nanoparticles for MRI contrast agents', Advanced Materials, 21(21), pp. 2133-2148. doi: 10.1002/adma.200802366.

Naqvi et al. (2010) 'Concentration-dependent toxicity of iron oxide nanoparticles mediated by increased oxidative stress', International Journal of Nanomedicine, p. 983. doi: 10.2147/ijn.s13244.

Nations, S., Wages, M., et al. (2011) 'Acute effects of $\mathrm{Fe} 2 \mathrm{O} 3$, $\mathrm{TiO} 2, \mathrm{ZnO}$ and $\mathrm{CuO}$ nanomaterials on Xenopus laevis', Chemosphere, 83(8), pp. 1053-1061. doi:

http://dx.doi.org/10.1016/j.chemosphere.2011.01.061.

Nations, S., Long, M., et al. (2011) 'Effects of ZnO nanomaterials on Xenopus laevis growth and development', Ecotoxicology and Environmental Safety, 74(2), pp. 203-210. doi: http://dx.doi.org/10.1016/j.ecoenv.2010.07.018.

Nieuwkoop, P. D. and Jacob Faber (1994) Normal Table of Xenopus Laevis (Daudin): A Systematical and Chronological Survey of the Development from the Fertilized Egg Till the End of Metamorphosis. New York: Garland Pub.

Palmiter, R. D. and Findley, S. D. (1995) 'Cloning and functional characterization of a mammalian zinc transporter that confers resistance to zinc.', The EMBO journal, 14(4), pp. 639-649. doi: 10.1002/j.1460-2075.1995.tb07042.x. 
Perelshtein, I. et al. (2015) 'The influence of the crystalline nature of nano-metal oxides on their antibacterial and toxicity properties', Nano Research, 8(2), pp. 695-707. doi: 10.1007/s12274-0140553-5.

Raeisi-Shahraki, R. et al. (2012) 'Structural characterization and magnetic properties of superparamagnetic zinc ferrite nanoparticles synthesized by the coprecipitation method', Journal of Magnetism and Magnetic Materials, 324(22), pp. 3762-3765.

Rivero, M. et al. (2016) 'Synthesis and structural characterization of ZnxFe3-xO4 ferrite nanoparticles obtained by an electrochemical method', Rsc Advances, 6(46), pp. 40067-40076. doi: 10.1039/c6ra04145k.

Sahoo, B. et al. (2013) 'Thermal and pH Responsive Polymer-Tethered Multifunctional Magnetic Nanoparticles for Targeted Delivery of Anticancer Drug', ACS Applied Materials \& Interfaces, 5(9), pp. 3884-3893. doi: 10.1021/am400572b.

Saide, K., Sherwood, V. and Wheeler, G. (2018) 'Paracetamol-induced liver injury modelled in Xenopus laevis embryos', Toxicology Letters.

Saquib, Q. et al. (2013) 'Zinc ferrite nanoparticles activate IL-1b, NFKB1, CCL21 and NOS2 signaling to induce mitochondrial dependent intrinsic apoptotic pathway in WISH cells', Toxicology and Applied Pharmacology. Elsevier Inc., 273(2), pp. 289-297. doi: 10.1016/j.taap.2013.09.001.

Tefft, B. et al. (2015) 'Cell Labeling and Targeting with Superparamagnetic Iron Oxide Nanoparticles', Journal of Visualized Experiments, 19(1105), p. e53099.

Thambiayya, K. et al. (2012) 'Functional role of intracellular labile zinc in pulmonary endothelium', Pulmonary Circulation, 2(4), pp. 443-451.

Tussellino, M. et al. (2015) 'Polystyrene nanoparticles affect Xenopus laevis development', Journal of Nanoparticle Research, 17(2). doi: 10.1007/s11051-015-2876-x.

Vance, M. et al. (2015) 'Nanotechnology in the Real World: Redeveloping the Nanomaterial Consumer Products Inventory', Beilstein Journal of Nanotechnology, 6(1), pp. 1769-1780.

Vu, M. M. T. et al. (2012) 'The DNLZ/HEP zinc-binding subdomain is critical for regulation of the mitochondrial chaperone HSPA9', 21(2), pp. 258-267. doi: 10.1002/pro.2012.

Wadhwa, R. et al. (2003) 'Targeting mortalin using conventional and RNA-helicase-coupled hammerhead ribozymes', 4(6), pp. 595-601. doi: 10.1038/sj.embor.embor855.

Wan, J. et al. (2012) 'Facile synthesis of zinc ferrite nanoparticles as non-lanthanide T1 MRI contrast agents', Journal of Materials Chemistry, 22, pp. 13500-13505.

Warheit, D. B., Sayes, C. M. and Reed, K. L. (2009) 'Nanoscale and fine zinc oxide particles: Can in vitro assays accurately forecast lung hazards following inhalation exposures?', Environmental Science and Technology, 43(20), pp. 7939-7945. doi: 10.1021/es901453p. 
Webster, C. et al. (2016) 'An early developmental vertebrate model for nanomaterial safety: bridging cell-based and mammalian toxicity assessment', Nanomedicine, 11(6), pp. 643-656. doi: 10.2217/nnm.15.219.

Wilkinson, N. and Pantopoulos, K. (2014) 'The IRP/IRE system in vivo: Insights from mouse models', Frontiers in Pharmacology, 5 JUL(July), pp. 1-15. doi: 10.3389/fphar.2014.00176.

Yamaji, S. et al. (2001) 'Zinc regulates the function and expression of the iron transporters DMT1 and IREG1 in human intestinal Caco-2 cells', FEBS Letters, 507(2), pp. 137-141. doi: 10.1016/S00145793(01)02953-2.

Ye, Q. et al. (2011) 'Bioinspired catecholic chemistry for surface modification', Chemical Society Reviews, 40(7). doi: 10.1039/c1cs15026j.

Zhang, D.-L. D., Ghosh, M. C. M. and Rouault, T. A. T. (2014) 'The physiological functions of iron regulatory proteins in iron homeostasis - an update', Frontiers in Pharmacology, 5, pp. 1-12. doi: 10.3389/fphar.2014.00124.

Zhang, Z. et al. (2017) 'Recent advances in magnetic targeting based on high magnetic field and magnetic particles', High Voltage, 2(4), pp. 220-232. doi: 10.1049/hve.2017.0082.

Zhu, S. et al. (2016) 'Evaluation of zinc-doped magnetite nanoparticle toxicity in the liver and kidney of mice after sub-chronic intragastric administration', Toxicol. Res. Royal Society of Chemistry, 5(1), pp. 97-106. doi: 10.1039/C5TX00292C.

\section{Supporting Information}



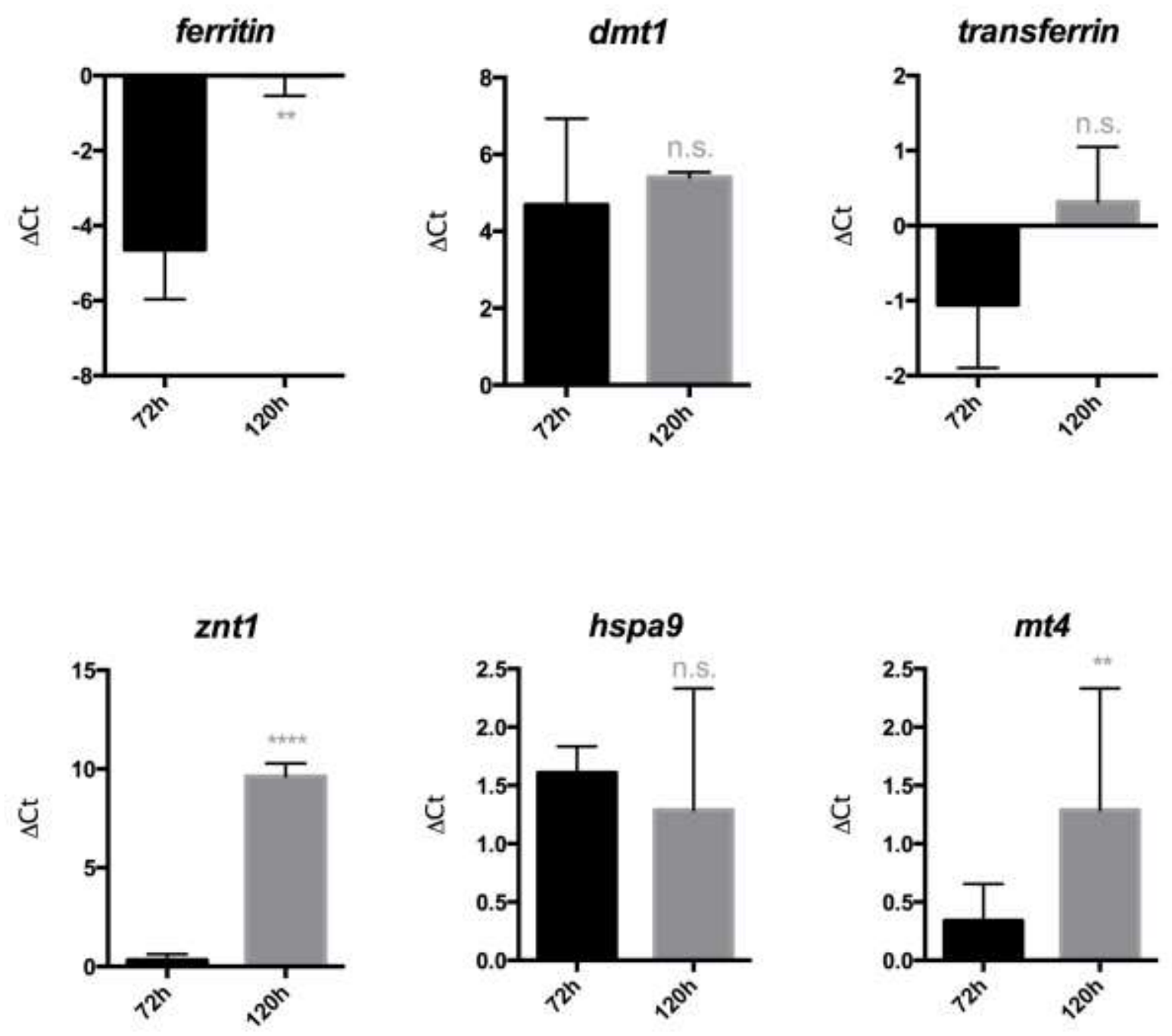

Figure SI1. Effect on the expression of different genes involved in iron and zinc metabolism in Xenopus laevis embryos during their development in different conditions $\left(72 \mathrm{~h}, 23^{\circ} \mathrm{C}\right)$ or $\left(120 \mathrm{~h}, 18^{\circ} \mathrm{C}\right) .(\mathrm{n}=7$ per group). * shows statistically significance compared with the control (Student's t-test, ${ }^{*} \mathrm{p}<0.05$, ${ }^{* *}$ $\left.p<0.01,{ }^{* * *} p<0.001,{ }^{* * * *} p<0.0001\right)$. 